\title{
Simultaneous Top-down Modulation of the Primary Somatosensory Cortex and Thalamic Nuclei during Active Tactile Discrimination
}

\author{
Miguel Pais-Vieira, ${ }^{1}$ Mikhail A. Lebedev, ${ }^{1,4}$ Michael C. Wiest, ${ }^{5}$ and Miguel A. L. Nicolelis ${ }^{1,2,3,4,6}$ \\ Departments of ${ }^{1}$ Neurobiology, ${ }^{2}$ Biomedical Engineering, and ${ }^{3}$ Psychology and Neuroscience and ${ }^{4}$ Center for Neuroengineering, Duke University, Durham, \\ North Carolina 27710, ${ }^{5}$ Neuroscience Program, Wellesley College, Wellesley, Massachusetts 02481, and ${ }^{6}$ Edmond and Lily Safra International Institute for \\ Neuroscience of Natal, 59066-060 Natal, Brazil
}

The rat somatosensory system contains multiple thalamocortical loops (TCLs) that altogether process, in fundamentally different ways, tactile stimuli delivered passively or actively sampled. To elucidate potential top-down mechanisms that govern TCL processing in awake, behaving animals, we simultaneously recorded neuronal ensemble activity across multiple cortical and thalamic areas while rats performed an active aperture discrimination task. Single neurons located in the primary somatosensory cortex (S1), the ventroposterior medial, and the posterior medial thalamic nuclei of the trigeminal somatosensory pathways exhibited prominent anticipatory firing modulations before the whiskers touching the aperture edges. This cortical and thalamic anticipatory firing could not be explained by whisker movements or whisker stimulation, because neither trigeminal ganglion sensory-evoked responses nor EMG activity were detected during the same period. Both thalamic and S1 anticipatory activity were predictive of the animal's discrimination accuracy. Inactivation of the primary motor cortex (M1) with muscimol affected anticipatory patterns in S1 and the thalamus, and impaired the ability to predict the animal's performance accuracy based on thalamocortical anticipatory activity. These findings suggest that neural processing in TCLs is launched in anticipation of whisker contact with objects, depends on top-down effects generated in part by M1 activity, and cannot be explained by the classical feedforward model of the rat trigeminal system.

\section{Introduction}

The rat's primary somatosensory cortex (S1) is the main cortical target of tactile information transmitted from the animals' facial whiskers upstream by the parallel pathways of the trigeminal system (Woolsey and Van der Loos, 1970). The feedforward "labeled line" model postulates that information generated by stimulation of each whisker ascends through parallel streams of hierarchical processing levels, within which distinctive neuronal clusters, named barrelets (in the brainstem), barreloids (thalamus), and barrels (cortex), isomorphically match the spatial organization of the whisker arrays on the rat's face (Woolsey and Van der Loos, 1970; Van Der Loos, 1976; Welker, 1976; Belford and Killackey, 1979).

More recently, several lines of evidence have suggested that, in addition to this bottom-up flow of information, somatosensory

\footnotetext{
Received April 3, 2012; revised Jan. 10, 2013; accepted Jan. 15, 2013.

Author contributions: M.P.-V. and M.A.L.N. designed research; M.P.-V. and M.C.W. performed research; M.P.-V., M.A.L., M.W., and M.A.L.N. analyzed data; M.P.-V., M.A.L., and M.A.L.N. wrote the paper.

This work was supported by NIH Grants R01DE011451, R01NS073125, and RC1HD063390, and by National Institute of Mental Health Award DP1MH099903 (M.A.L.N.). The content is solely the responsibility of the authors and does not necessarily represent the official views of the National Institutes of Health. We thank Eric Thomson for his thoughtful comments on this manuscript, Laura Oliveira and Susan Halkiotis for miscellaneous assistance, Jim Meloy for outstanding electrode manufacturing, and Zheng Li and Solaiman Shokur for help with animation.

Correspondence should be addressed to Miguel A. L. Nicolelis, Box 3209, Department of Neurobiology, Duke University, Durham, NC 27710. E-mail: nicoleli@neuro.duke.edu.

DOI:10.1523/JNEUROSCI.1659-12.2013

Copyright $\odot 2013$ the authors $\quad 0270-6474 / 13 / 334076-18 \$ 15.00 / 0$
}

processing is significantly affected by top-down modulations that reflect past experience (Nicolelis and Chapin, 1994; Ghazanfar and Nicolelis, 1997; Krupa et al., 1999; Gutierrez et al., 2010; Wiest et al., 2010; Ego-Stengel et al., 2012), ongoing motor activity (Fanselow et al., 2001; Brecht et al., 2004; Urbain and Deschenes, 2007b; Lee et al., 2008; Hill et al., 2011), reward expectations (Pantoja et al., 2007), and interhemispheric coordination (Shuler et al., 2001; Wiest et al., 2005).

During the past decade, we have studied the physiological properties of ensembles of cortical and thalamic neuronal ensembles while rats actively discriminated the width of an aperture (Krupa et al., 2001, 2004). Using this task, we showed previously that the activity of S1 neurons in awake rats is fundamentally different depending on whether an animal actively explores tactile stimuli with their whiskers or whether these mechanical stimuli are delivered passively to their vibrissae (Fanselow et al., 2001; Krupa et al., 2004). Moreover, during active tactile exploration, a significant number of S1 neurons modulate their firing rates before the whisker contact with the stimulus (Krupa et al., 2004; Wiest et al., 2010). Further experiments have shown that this anticipatory S1 activity is refined as animals learn a tactile discrimination task (Wiest et al., 2010).

Several lines of evidence support the hypothesis that such anticipatory firing modulations in S1 may be produced, among other sources, by afferents from the primary motor cortex (M1) (Lee et al., 2008), a cortical area that has a fundamental role in active exploratory behavior, particularly in whisker positioning 
(Hill et al., 2011). This hypothesis derives from the notion that centrally generated corollary discharges originating in M1 would have the function of modulating sensory neurons at cortical and subcortical levels before the arrival of ascending tactile information generated by peripheral sensory stimuli (Sperry, 1950).

In the present study, we investigate the role of M1 in the formation of anticipatory activity in multiple thalamocortical loops (TCLs). To achieve this goal, we recorded from neuronal ensembles in M1 and S1 or M1 and both the ventral posterior medial nucleus (VPM) and posterior (POM) nucleus of the thalamus while rats performed the same active aperture discrimination task. Additionally, we inactivated M1 with muscimol while rats were performing the aperture discrimination task.

\section{Materials and Methods}

Subjects and active tactile discrimination task. All animal procedures were performed in accordance with the National Research Council's Guide for the Care and Use of Laboratory Animals and were approved by the Duke University Institutional Animal Care and Use Committee. Long Evans female rats $(n=23)$ weighing between 250 and $350 \mathrm{~g}$ were used in all experiments. The animals were mildly water deprived and trained to perform a behavioral discrimination task as described previously (for a task description, see Fig. 2B) (Krupa et al., 2001). Briefly, this task required animals to discriminate between a wide or narrow aperture to receive a water reward. At the beginning of each session, animals were placed in the behavioral box compartment called the outer chamber, where they waited for the central door to open and allow access to the second compartment, the inner chamber. After the animal entered the inner chamber, it had to use its whiskers to touch the edges of an aperture, formed by computer-controlled bars (hereafter referred to as the discrimination bars). The width of this aperture varied from trial to trial. Rats had to judge the aperture diameter and then nose poke the center of the front wall (see Fig. $2 \mathrm{~B}$ ). Animal presence near the discrimination bars and near the front wall was detected by a photobeam. The nose poke in the inner chamber opened two water reward pokes located in the outer chamber from which the animal had to select one. The reward poke on the right corresponded to the wide aperture, whereas the poke on the left corresponded to the narrow aperture. As the animal chose a reward poke, the door separating the inner and outer chambers closed. Correct responses were rewarded by $50 \mu \mathrm{l}$ water rewards, after which both reward pokes were closed. Incorrect responses were followed by their immediate closing. The aperture was set for a new trial $5-8 \mathrm{~s}$ after the reward pokes were closed.

The animal's performance was measured by calculating the percentage of trials performed correctly during a session. The average number of trials per session $(n=101.5 \pm 3.0)$ and the mean time spent $(n=270 \pm$ $0.8 \mathrm{~ms}$ ) between the door and discrimination bars were used as measures of motor performance. High-resolution video recordings were conducted in sessions separate from the ones where neural activity was recorded using a high-speed camera (SI-1300M-H-CL; Silicon Imaging) to assess the animal's behavior quantitatively. Video analysis of task performances was conducted using in house semiautomated software. The amount of time that the animal's whiskers were in contact with the discrimination bars was measured and compared across conditions (control, saline, and muscimol conditions) for 12 sessions (four for each condition). Additionally, high-resolution video recordings of 24 sessions presented previously (Wiest et al., 2010) were reanalyzed to determine the distribution of early whisker contacts [i.e., preceding the beam break (BB)].

Multielectrode implants. After the animals were trained in the behavioral task, microelectrodes were surgically implanted in multiple cortical and thalamic areas. The animals were given access to water for a period of at least $24 \mathrm{~h}$ before surgery, and for at least $7 \mathrm{~d}$ after the surgery. Cannulamicroelectrode bundles and/or arrays of electrodes were implanted in the M1, S1, VPM, and POM. Six animals received unilateral implants in both M1 and S1. The other six animals were implanted in three areas: M1, VPM, and POM. Three animals had bilateral implants in M1 and S1.
Craniotomies were made and arrays lowered at the following stereotaxic coordinates for each area: $\mathrm{S} 1,-3.0 \mathrm{~mm} \mathrm{AP,} 5.5 \mathrm{~mm} \mathrm{ML},-0.2 \mathrm{~mm}$ $\mathrm{DV} ; \mathrm{M} 1,+2.0 \mathrm{~mm} \mathrm{AP},+2.0 \mathrm{~mm} \mathrm{ML},-1.5 \mathrm{~mm}$ DV; VPM, $-3.5 \mathrm{~mm}$ AP, $3.3 \mathrm{~mm} \mathrm{ML},-5.2 \mathrm{~mm} \mathrm{DV}$; POM, $-3.5 \mathrm{~mm} \mathrm{AP,} 2.1 \mathrm{~mm} \mathrm{ML},-5.2$ $\mathrm{mm}$ DV. Recording sites were histologically verified by comparing cresyl-stained $60 \mu \mathrm{m}$ coronal brain sections with reference anatomical planes (Paxinos and Watson, 1998).

To provide control data for potential peripheral afferent activation during the anticipatory period, five additional rats were implanted: two were implanted bilaterally in the trigeminal ganglion (TG; $-1.5 \mathrm{~mm} \mathrm{AP}$, $\pm 2.5 \mathrm{~mm} \mathrm{ML}$ ) with movable electrode bundles, and three rats were implanted unilaterally in the trigeminal ganglion and in VPM and S1 (contralateral to the implanted trigeminal ganglion). TG electrodes were implanted at the depth of $10.4 \mathrm{~mm}$ from the cortical surface and were then gradually advanced (in 63-250 $\mu \mathrm{m}$ steps) during the recording sessions as described previously (Nicolelis et al., 1995; Wiest et al., 2010). TG activity was identified at depths of $\sim 10.4-11.2 \mathrm{~mm}$ by noting clear whisker-evoked responses in the audio of the spiking activity and clear short-latency sensory-evoked responses.

Electrophysiological recordings. A multineuronal acquisition processor (64 channels; Plexon) was used to record neuronal spikes as described previously (Nicolelis et al., 1999). Briefly, neural signals were recorded differentially, amplified (20,000-32,000×), filtered (filtering band between $400 \mathrm{~Hz}$ and $5 \mathrm{kHz}$ ), and digitized at $40 \mathrm{kHz}$. Up to four single neurons per recording channel were sorted online (Sort Client 2002; Plexon). Online sorting was validated offline using Offline Sorter 2.8.8 (Plexon) according to the following cumulative criteria: (1) signal-tonoise ratio $>2.5$ (as verified on the oscilloscope screen); (2) $<0.1 \%$ of interspike intervals (ISIs) smaller than $1.0 \mathrm{~ms}$; (3) stereotypy of waveform shapes, as determined by a waveform template algorithm and principal component analysis. These cumulative criteria were complemented with inspection of metrics of the quality of single unit isolation in behaving animals (J3, Davis-Bouldin, $F$, and pseudo-F) (Nicolelis et al., 2003).

In S1, VPM, and POM, microelectrodes were lowered from initial positions of $-0.2 \mathrm{~mm},-5.2$, and $-5.2 \mathrm{~mm}$, respectively. Steps of at least $62.5 \mu \mathrm{m}$ were used to move the microelectrodes after a similar number of control, saline, and muscimol sessions were recorded (typically two), or when a very small number of units were recorded in one session. It is possible that the same units were repeatedly recorded by the same electrode in different sessions. However, we did not assume that the same units were recorded on each channel on different days because muscimol inactivation very often was associated with masking and unmasking of neurons in M1 and the other areas recorded, making it difficult to judge if a waveform reappearing after a muscimol session belonged to the same or different neuron.

Inactivation with muscimol. To inactivate M1, muscimol (500 ng in 500 $\mathrm{nl}$ of saline) or saline $(500 \mathrm{nl})$ was slowly injected unilaterally with a microperfusion precision pump (Harvard Apparatus) for a period of 4 min under isofluorane anesthesia or in awake, behaving animals in an open field (in four animals). This dose of muscimol inactivates an injected cortical volume for 6-8 h (Martin, 1991; Krupa et al., 1999; Shuler et al., 2001, 2002). The inactivation effect was confirmed by an absence of action potentials on the electrodes surrounding the injected area.

The sequence of control, saline, and muscimol sessions was changed randomly in the same animals to avoid any possible bias. Video recordings of four sessions in each condition were made to assess possible motor impairments due to muscimol effects in M1.

Bilateral facial nerve lesions combined with EMG recordings. To test whether whisker movements were required for anticipatory activity to occur, we performed bilateral facial nerve lesions in three rats. The nerve cut procedure was performed as described previously (Krupa et al., 2001). Briefly, rats were anesthetized with ketamine $(100 \mathrm{mg} / \mathrm{kg})$ and xylazine $(10 \mathrm{mg} / \mathrm{kg})$. The facial fur posterior to the whisker pad was shaved. An incision was made in the skin $\sim 3-4 \mathrm{~mm}$ posterior to whisker E1. The soft tissue below the skin was carefully dissected to expose the facial nerve. A small loop of 3-0 surgical suture was then tied tightly around the nerve and secured with a small hemostat. A portion of $\sim 2$ $\mathrm{mm}$ of the nerve was then cut, and each cut end was crushed with a small hemostat. To control for muscle contractions possibly remaining after 
these facial nerve lesions, bundles of four electrodes for EMG recordings were implanted in the whisker pad. The wires were passed subcutaneously through a catheter and exited at the top of the scalp near the connector used for neuronal recordings. The wound was then closed with a suture, and the procedure was repeated on the opposite side of the face. Rats were given $5 \mathrm{~d}$ of postsurgical recovery with access to food and water ad libitum.

Data analysis. Neuronal data obtained from a total of 151 recording sessions were processed and analyzed using NeuroExplorer (version 3.266; NEX Technologies) and custom scripts written in MATLAB (7.9.0; MathWorks). A trial was defined as the period from -2.0 to $2.0 \mathrm{~s}$ relative to the time when the rat broke the photobeam at the discrimination aperture (see Fig. $2 \mathrm{~B}$ ). The beam break was defined as time $0 \mathrm{~s}$ in all analyses and figures presented. Anticipatory activity was defined as neuronal modulations occurring in the period of -0.5 to $0 \mathrm{~s}$. Anticipatory activity was further divided according to early anticipatory (from -0.5 to $-0.2 \mathrm{~s}$ ) and late anticipatory (from -0.2 to $0 \mathrm{~s}$ ). Statistical significance of neural responses was evaluated using a method based on cumulativesummed spike counts (Wiest et al., 2005; Gutierrez et al., 2006). The period of -1.5 to $-0.5 \mathrm{~s}$ was used as a baseline in this test. Depending on the firing rate change, response types were classified as "increased," "decreased," or "multiphasic" (i.e., a combination of rate increases and decreases). The proportion of single units presenting each type of firing modulation was compared using $\chi^{2}$ tests. For statistical tests with two comparisons (e.g., control versus saline and control versus muscimol), we used $\alpha=0.025$; otherwise we used $\alpha=0.05$. Response magnitude was defined as the average difference in firing rate between the period of interest and the background. Response duration was defined as the time interval during which a unit's firing significantly deviated from the background. Comparisons of characteristics of neural responses for different conditions were performed using nonparametric tests (Mann-WhitneyWilcoxon or Kruskal-Wallis).

Principal component analysis was applied to normalized data (divided by the maximum value) for all the parameters examined and calculated using a custom MATLAB script. The principal components that cumulatively explained the largest portion of variance (at least $80 \%$ ) were selected, and the loadings of each variable in these components were further analyzed. Color-coded plots of each neuron's activity were normalized by dividing each value by the maximum firing rate.

In the offline analysis, EMG signals were high-pass filtered with a bandpass Butterworth filter run sequentially in the forward and reverse directions. A cutoff frequency of $50 \mathrm{~Hz}$ was used. Rectified EMG signals were then calculated as the absolute value, $|\Delta \mathrm{EMG}|$. EMG events were considered as periods of EMG activity with increases of more than three SDs from the mean.

Analysis of patterns of increased and decreased neuronal activity. To characterize the neuronal activity patterns across multiple thalamocortical loops during the performance of an active tactile task, we pooled data from all subjects used in control studies, including those that received saline injections. We also included 261 units recorded in S1 from seven animals injected with $500 \mathrm{nl}$ of saline in S1 contralateral to the recording site. Neuronal data from each trial were time-aligned when the rats entered the aperture. Neuronal discharges were counted in sequential $10 \mathrm{~ms}$ time bins and normalized to the maximum value across all bins. The normalized values were then smoothed with a moving average window of $250 \mathrm{~ms}$ (since this was a typical duration of neuronal responses in the behavioral task). These smoothed firing rates were then used to describe activity modulations (both increased and decreased responses) in multiple cortical areas and thalamic structures, and to relate these activity changes to specific task events. In particular, we analyzed responses occurring before, during, and after whisker contact with the tactile stimulus.

Linear regression for tactile performance prediction. Linear regression was used to analyze the relationship between neuronal activity and behavioral parameters, such as task performance accuracy. Animals' performance accuracy was also compared with their speed. First, we examined increased responses that occurred in the period between -250 and $0 \mathrm{~ms}$ relative to the aperture area entrance. This period corresponded to the rat going from the central door to the discrimination bars. Antic- ipatory neural activity increases were concentrated in this period. The pattern of inhibited firing was not used in this analysis because this type of modulation was distributed across the whole length of a trial. For each session, we first identified significant deviations in firing rate from baseline for all units during the period between -250 and $0 \mathrm{~ms}$. The average of these values was considered as the response onset for that session. Animal speed was derived from the average time that it took the rat to get from the door to the discrimination bars. Sessions where the animals got trapped in the door between the reward and discrimination chamber or performed close to chance ( $<60 \%$ of correct responses) were not used in the linear regression analysis.

Neural events of interest. Trial-by-trial analysis of neuronal ensemble activity was conducted for the compound activity calculated for all S1 units recorded in a single session. This approach allowed us to test whether the effects of cortical inactivation could be observed in the overall activity of the entire recorded ensemble (including the units whose modulations alone were not statistically significant). Each trial was divided into $50 \mathrm{~ms}$ bins, and the interval -2 to $-0.5 \mathrm{~s}$ was used to build a baseline probability distribution. Since the number of bins that constituted the baseline was fairly small $(N=30$, for a total period of $1.5 \mathrm{~s})$, we analyzed only the first bin in the anticipatory period -0.5 to $0 \mathrm{~s}$ where statistically significant $(p \leq 0.05)$ differences in spike counts were found. As our neuronal baseline firing distribution was built only with the bin counts from a specific trial, it cannot be considered a true baseline distribution. Consequently, we did not consider bin counts with a probability $p \leq 0.05$ to be significant, but instead we used the term "neural event of interest" (NEI).

A total of 57 sessions from data recorded across S1 layers were analyzed. Ensembles with less than six units were not analyzed because a very small number of cells could potentiate increases in the probability of anticipatory NEIs even with small variations in activity. We set the value of 6 as the lowest number of units since it allowed us to have at least two units with every type of response sampled (increased, decreased, multiphasic), making it more likely that a large number of sessions could be analyzed, while extreme variations in the firing pattern of one unit would have little or no effect. For the comparison of variation in the ensemble firing rate before and after whiskers contacted the stimulus, we used the average values for the periods $-500-0 \mathrm{~ms}$ and $0-300 \mathrm{~ms}$. The interval of $-500-0 \mathrm{~ms}$ was used since the overall ensemble activity suggested that changes started during this interval.

To perform the analysis, we first identified which trials contained NEIs during the early or late anticipatory period (intervals of -500 to -200 $\mathrm{ms}$ and -200 to $0 \mathrm{~ms}$, respectively). Second, for each "early" or "late" NEI trial, we calculated the difference between the average of the firing rate before and after the whiskers contacted the tactile discriminanda. This allowed us to quantify the variation in the ensemble mean firing rate before and after the tactile discrimination when NEIs were present in early or late periods. Last, we compared the variation in each condition using a Mann-Whitney-Wilcoxon test.

\section{Results}

A total of 15 rats were implanted with microelectrode arrays and an injection cannula for muscimol delivery. Single-unit activity was recorded simultaneously in S1 and M1, or in M1, VPM, and POM while rats performed an active aperture discrimination task (Krupa et al., 2001; Wiest et al., 2010) before, during, and after pharmacological inactivation of M1 ipsilateral to the recording sites.

A total of 2575 single S1, VPM, and POM units were recorded in 120 behavioral sessions from the microelectrodes implanted across four different regions (see Figs. 2-4). Figure 1 illustrates examples of the quality of cluster separation $(A)$ waveforms, $(B)$ ISI distribution $(C, D)$, as well as cluster-related statistics (Nicolelis et al., 2003) (J3, $2.939 \pm 0.08$; pseudo-F, 49,773 \pm 2507 ; Davies-Bouldin, $0.1981 \pm 0.01 ; F, 1.725 \pm 0.02$; Fig. $1 E-H)$. All of these measurements confirmed the high quality of single unit isolation obtained in each of the sampled brain structures. The 

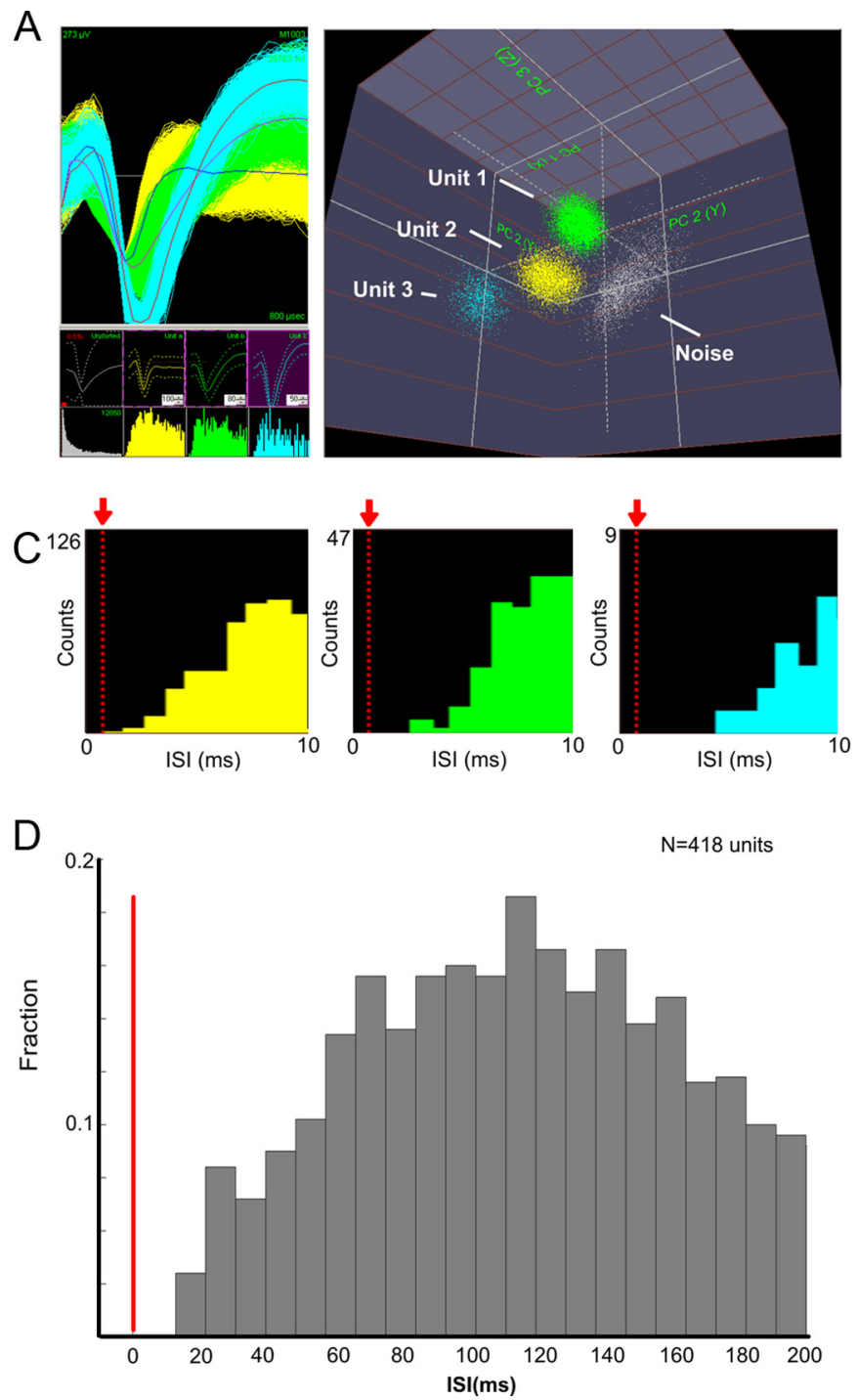

B

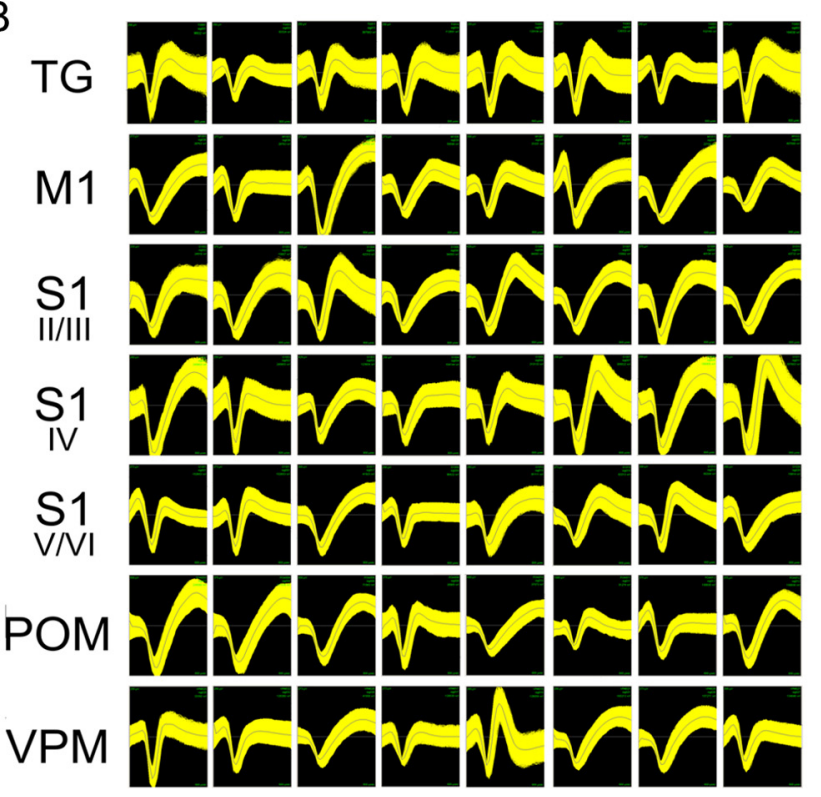

E
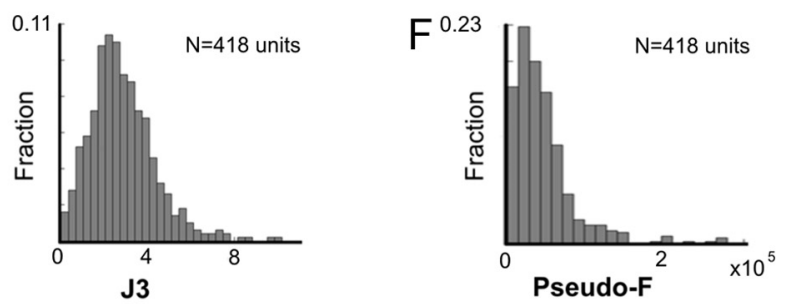

G

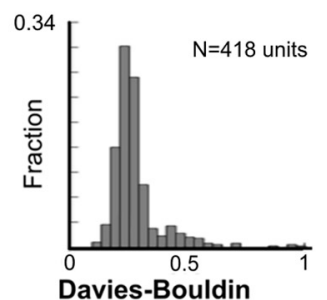

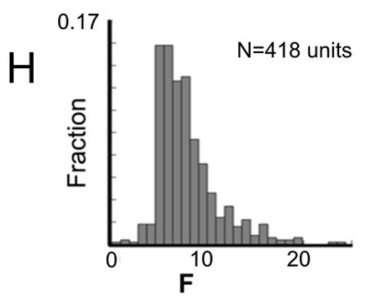

Figure 1. Cluster separation and waveform quality in recordings. $\boldsymbol{A}$, Typical examples of single unit waveforms and cluster separation (3D) recorded. $\boldsymbol{B}$, Examples of waveforms from single units recorded in all regions studied. $\boldsymbol{C}$, ISIs of Units $1-3$ presented in $\boldsymbol{A}$. The red arrows indicate the refractory period. Note the absence of spike counts in the refractory period. $\boldsymbol{D}$, Distribution of average ISls for single units recorded in all control sessions. $\boldsymbol{E}-\boldsymbol{H}$, Distributions of single unit statistics for all control sessions.

proportion of units recorded in each region was as follows: $39.42 \%$ in S1 ( $n=1015$ units), $19.57 \%$ in VPM ( $n=504$ units), $17.94 \%$ in POM ( $n=462$ units), and $23.07 \%$ in M1 $(n=594$ units). In the $\mathrm{S} 1,40.99 \%$ ( $n=416$ units) were recorded from the supragranular layers, $30.44 \%$ ( $n=309$ units) were recorded from the granular layer, and $28.56 \%(n=290$ units $)$ were recorded from the infragranular layers. Additionally, we recorded single units ( $n=31$ units) and multiunits ( $n=705$ multiunits) from the TG. Note that as the electrode arrays were not moved every session, it is possible that the number of single units could be slightly smaller than values reported above. We estimate that $\sim 20 \%$ of the neurons recorded remained the same across different sessions.

Overall, statistically significant modulations of firing rates were found in a large proportion (75\%) of neurons in all conditions and regions tested (Table 1). Specifically, we found patterns of concurrent increased and decreased neuronal activity that varied across different layers of S1 and thalamic nuclei (VPM and $\mathrm{POM}$ ). In the control condition, anticipatory firing modulations were observed in $40.19 \%$ of the S1 units, $49.67 \%$ of the VPM units, and $37.93 \%$ of the POM neurons recorded in this study.
The magnitude of anticipatory firing in S1 was $2.72 \pm 0.1$ spikes per trial, and its duration was $195.4 \pm 14.62 \mathrm{~ms}$. In VPM, the magnitude of the anticipatory firing was $2.61 \pm 0.3$ spikes per trial, and its duration was $247.4 \pm 19.51 \mathrm{~ms}$. In POM, the magnitude of the anticipatory firing was $2.4 \pm 0.2$ spikes per trial, and its duration was $184.0 \pm 32.58 \mathrm{~ms}$. Such modulation in neuronal firing frequently started several hundred milliseconds before the animals' facial whiskers made any physical contact with the tactile stimulus (Figs. 2A, 3). Characteristic examples of these anticipatory firing modulations in different $\mathrm{S} 1$ layers and VPM and POM nuclei can be observed in the peristimulus time histograms (PSTHs) depicted in Figure $2 \mathrm{~A}$. Note that multiple increases and decreases of cortical and thalamic firing occur before the animals break the infrared beam and touch the edges of the bar with their facial whiskers (Fig. 2B). Different colors in Figure $2 B$ schematically illustrate the relationship between the behavioral task and the different analysis periods. The anticipatory epoch corresponds to the period before the whiskers make contact with the discrimination bars (light blue), while the discriminatory period (green) corresponds to the period immediately after the whiskers touch the target bars. The observed cortical and thalamic firing modulations were 
Table 1. Proportions and type of firing modulations by region

\begin{tabular}{|c|c|c|c|c|c|c|c|}
\hline & Modulated (fraction) & Increased (fraction) & Decreased (fraction) & Multiphasic (fraction) & $\chi^{2}$ & df & $p$ \\
\hline \multicolumn{8}{|l|}{ Supragranular } \\
\hline Control & 0.81 & 0.41 & 0.26 & 0.34 & & & \\
\hline Saline & 0.65 & 0.48 & 0.26 & 0.26 & 1.63 & 2 & 0.4426 (n.s.) \\
\hline Muscimol & 0.67 & 0.46 & 0.20 & 0.34 & 0.84 & 2 & 0.657 (n.s.) \\
\hline \multicolumn{8}{|l|}{ Granular } \\
\hline Control & 0.83 & 0.64 & 0.11 & 0.25 & & & \\
\hline Saline & 0.77 & 0.52 & 0.17 & 0.32 & 2.63 & 2 & 0.2685 (n.s.) \\
\hline Muscimol & 0.69 & 0.61 & 0.17 & 0.22 & 1.29 & 2 & 0.5247 (n.s.) \\
\hline \multicolumn{8}{|l|}{ Infragranular } \\
\hline Control & 0.82 & 0.27 & 0.27 & 0.46 & & & \\
\hline Saline & 0.83 & 0.47 & 0.18 & 0.35 & 4.02 & 2 & 0.134 (n.s.) \\
\hline Muscimol & 0.75 & 0.56 & 0.05 & 0.39 & 18.9 & 2 & $<0.0001^{* * *}$ \\
\hline \multicolumn{8}{|l|}{ POM } \\
\hline Control & 0.78 & 0.47 & 0.21 & 0.32 & & & \\
\hline Saline & 0.74 & 0.51 & 0.21 & 0.28 & 0.56 & 2 & 0.7558 (n.s.) \\
\hline Muscimol & 0.73 & 0.54 & 0.15 & 0.31 & 1.91 & 2 & 0.3848 (n.s.) \\
\hline \multicolumn{8}{|l|}{ VPM } \\
\hline Control & 0.82 & 0.29 & 0.21 & 0.50 & & & \\
\hline Saline & 0.74 & 0.33 & 0.29 & 0.38 & 3.79 & 2 & 0.1503 (n.s.) \\
\hline Muscimol & 0.72 & 0.47 & 0.30 & 0.22 & 21.42 & 2 & $<0.0001^{* * *}$ \\
\hline
\end{tabular}

not restricted to one specific task period, but instead occurred during many different time epochs. Figure 2, $C$ and $D$, depicts the average performance and average number of trials for each condition studied (see below for detailed description).

Each panel of Figure 3 shows the normalized firing activity (relative to the maximum firing rate of each neuron) of all of the cortical and thalamic neurons recorded during the execution of the tactile discrimination task in control conditions, and after saline or muscimol injections in M1. Continuous changes in neuronal activity occurred before and after stimulus contact within all cortical and thalamic regions sampled during the animals' performance of the tactile discrimination task (Figs. 3, 4). Anticipatory activity, i.e., before any whisker contact with aperture edges, was represented by both increases and/or decreases in neuronal firing. In VPM and granular layer of S1, anticipatory activity was mostly associated with a decrease in firing. In POM and S1 infragranular layers, the pattern of anticipatory activity followed the opposite trend (i.e., firing rate increased immediately before tactile discrimination). Based on previous published studies from our laboratory (Krupa et al., 2004; Wiest et al., 2010), the presence of these different patterns of neuronal firing modulations, within and between different structures, suggested that cortical and thalamic neuronal anticipatory firing was fundamental for task performance. To demonstrate the relationship between the animal's behavior during a trial and the diversity of neuronal firing modulations observed across multiple cortical and thalamic structures sampled in this study, Figure $4 \mathrm{~A}$ depicts neuronal activity rank ordered by time, from -2.0 to $2 \mathrm{~s}$. The multiple PSTHs presented show peaks of increased and decreased activation in all cortical and thalamic regions throughout a trial. The sequential order by which these peaks appear suggests the hypothesis that active tactile discrimination relies significantly on top down effects that cannot be explained by the classic feedforward model of tactile information processing. This hypothesis is supported by the finding that TG neurons (Fig. $4 A$, bottom PSTH) only start modulating their firing rate after the rat's whiskers touch the aperture edges. In Figure $4 B$, the fraction of neu- rons with significant increases or decreases in responses is shown by cortical area or thalamic nucleus. As noted above, virtually identical patterns of anticipatory firing activity occurred in VPM and in the granular layer of S1. Conversely, the patterns of anticipatory firing increases in the POM, M1, and infragranular layers of S1 also look similar.

Histological analysis (Fig. 5A) was used to locate the thalamic recordings sites. Different functional compartments (Fig. 5B), coincident with different depths of recording, have been described previously for the VPM, namely, the "head" and "core" of the barreloids (Urbain and Deschenes, 2007a). Thus, we further investigated whether neural anticipatory modulations during tactile discrimination were restricted to a specific VPM depth. Solely for this analysis, we pooled data from all the control and saline sessions reported here and added 99 units recorded in VPM and 168 units recorded from POM $(n=4$ animals in 10 sessions from a different study that used the same task; these animals were either control subjects or injected with 500nl of saline in S1). Figure $5 A-C$ illustrates that the depths of the recordings coincident with the head (starting at $-5.2 \mathrm{~mm}$ ) and core (starting at $-5.4 \mathrm{~mm}$ ) of the barreloids in VPM are associated with fundamentally different physiological properties, as reported previously in anesthetized animals (Urbain and Deschenes, 2007a). The region of the head of the barreloids was characterized by anticipatory activity coincident with the major periods of increased activity in POM, M1, and S1 infragranular layers. On the other hand, the core of the barreloids was coincident with the pattern of decreased-increased-decreased activity found in layer IV of S1 (see Fig. 5C). These two subregions of the VPM nucleus exhibited different proportions of anticipatory firing increases (VPM head, 31 of 64 units; VPM core, 54 of 256 units; $\chi^{2}=18.25$; $\mathrm{df}=1 ; p<0.0001)$ and decreases (VPM head, 4 of 64 units; VPM core, 107 of 256 units; $\chi^{2}=29.2$; df $\left.=1 ; p<0.0001\right)$. Despite these differences, cells with anticipatory increased activity were found at all depths studied.

Last, due to the proximity of POM and VPM head regions (Fig. 5A,B), we compared the physiological properties of neu- 

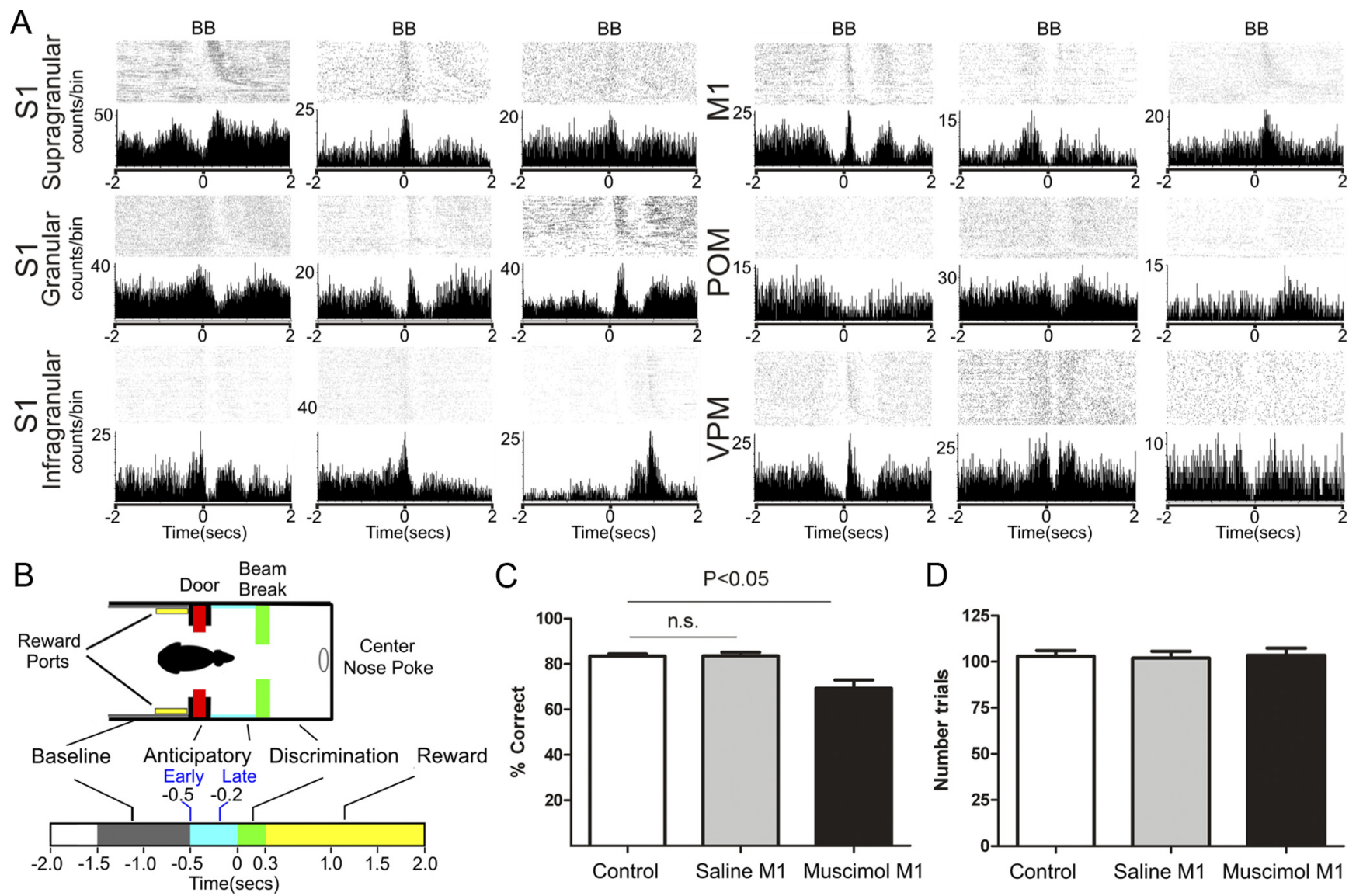

Figure 2. Anticipatory activity across the multiple thalamocortical loops of the rat trigeminal system. $\boldsymbol{A}$, Examples of single unit responses recorded across the thalamocortical loops as seen in PSTHs centered around the beam break (time 0 ) around which whiskers make contact with the aperture edges. Notice that periods of increased or decreased anticipatory firing activity occur frequently before the whiskers make contact with the tactile stimulus ( 0 s). All cortical areas (S1 and M1) and thalamic nuclei (VPM and POM) exhibit such anticipatory firing. $\boldsymbol{B}$, The top shows a schematic of the behavioral chamber, whereas the bottom utilizes different colors to indicate the different epochs of the behavioral task. All times are referenced to the BB in the discrimination bars $(0 \mathrm{~s})$. A trial starts at $-2 \mathrm{~s}$. Note that baseline activity is coincident with the period before the rat crosses the door (in red) that separates the outer and inner chambers. The anticipatory period corresponds to the epoch before the whiskers make contact with the discrimination bars (light blue). The discrimination period includes the time after the beam break and center nose poke (green). The reward period includes the time between the center nose poke, the decision, and the reward port nose poke (yellow). Anticipatory analysis includes an early (from -0.5 to $-0.2 \mathrm{~s}$ ) and a late period (from -0.2 to $0 \mathrm{~s}$ ). These two periods were determined based on the distribution of neural responses (for details, see Materials and Methods). $\boldsymbol{C}$, Percentage of correct discrimination trials in control or after saline or muscimol injections in M1. D, Number of trials performed in each condition. Although M1 inactivation impaired tactile discrimination, the number of trials performed or speed of performance were not affected, suggesting that no gross motor impairments were present. Error bars indicate SEM.

rons recorded from these two areas. Clear differences were found in the proportion of significant increased responses in POM in the anticipatory period corresponding to the rat entering the inner chamber (VPM head, 6 of 31 responses; POM, 50 of 125 responses; $\left.\chi^{2}=3.75 ; \mathrm{df}=1 ; p=0.05\right)$, in the magnitude of decreased neural activity (VPM head, $1.68 \pm 0.1$ spikes per trial; POM, $1.44 \pm 0.1$ spikes per trial; Mann-Whitney $U=4616$; $p=$ 0.0187 ), and in the duration of increased neural responses (VPM head, $153.0 \pm 25.31 \mathrm{~ms}$; POM, $182.4 \pm 9.94 \mathrm{~ms}$; Mann-Whitney $U=12540 ; p=0.05)$. Together, these results show that distinct compartments associated with the head and core of the VPM exhibit anticipatory increased and decreased neural activity before whisker contact with a discriminanda.

Overall, cortical and thalamic neuronal firing preceding the tactile stimulus could have originated from three possible sources: (1) whiskers contacting chamber walls or floor surface during the interval from the door opening and the aperture beam break, (2) whisker movements producing sensory reafference that triggered thalamic activity, or (3) top down neuronal afferents that induced anticipatory firing unrelated to whisker contact or movement. The first two possibilities have been mostly ruled out in previous studies conducted in our laboratory that demonstrated that whisker movements or early whisker contacts with the chamber walls are not the basis for anticipatory activity observed in S1 (Krupa et al., 2001, 2004; Wiest et al., 2010). To rule out these possibilities once and for all, we conducted two additional control experiments.

Neurons in the trigeminal ganglion are not modulated before any contact with the tactile stimulus

To control for the possibility of early whisker contacts with the chamber walls or floor, we simultaneously recorded neuronal activity from TG, S1, and VPM in the same subjects while rats performed the same tactile discrimination task. TG is the main recipient of primary afferent inputs from the whiskers, and thus the presence of neuronal responses in this ganglion provides a very reliable indicator of any mechanical displacement of the animal's facial vibrissae. Figure $6 \mathrm{~A}$ depicts a sample of PSTHs to illustrate the characteristic TG neuron firing modulations during execution of the tactile discrimination task. Analysis of these TG neurons' firing rate modulations revealed three main periods of increased activation corresponding to whisker contacts with the 


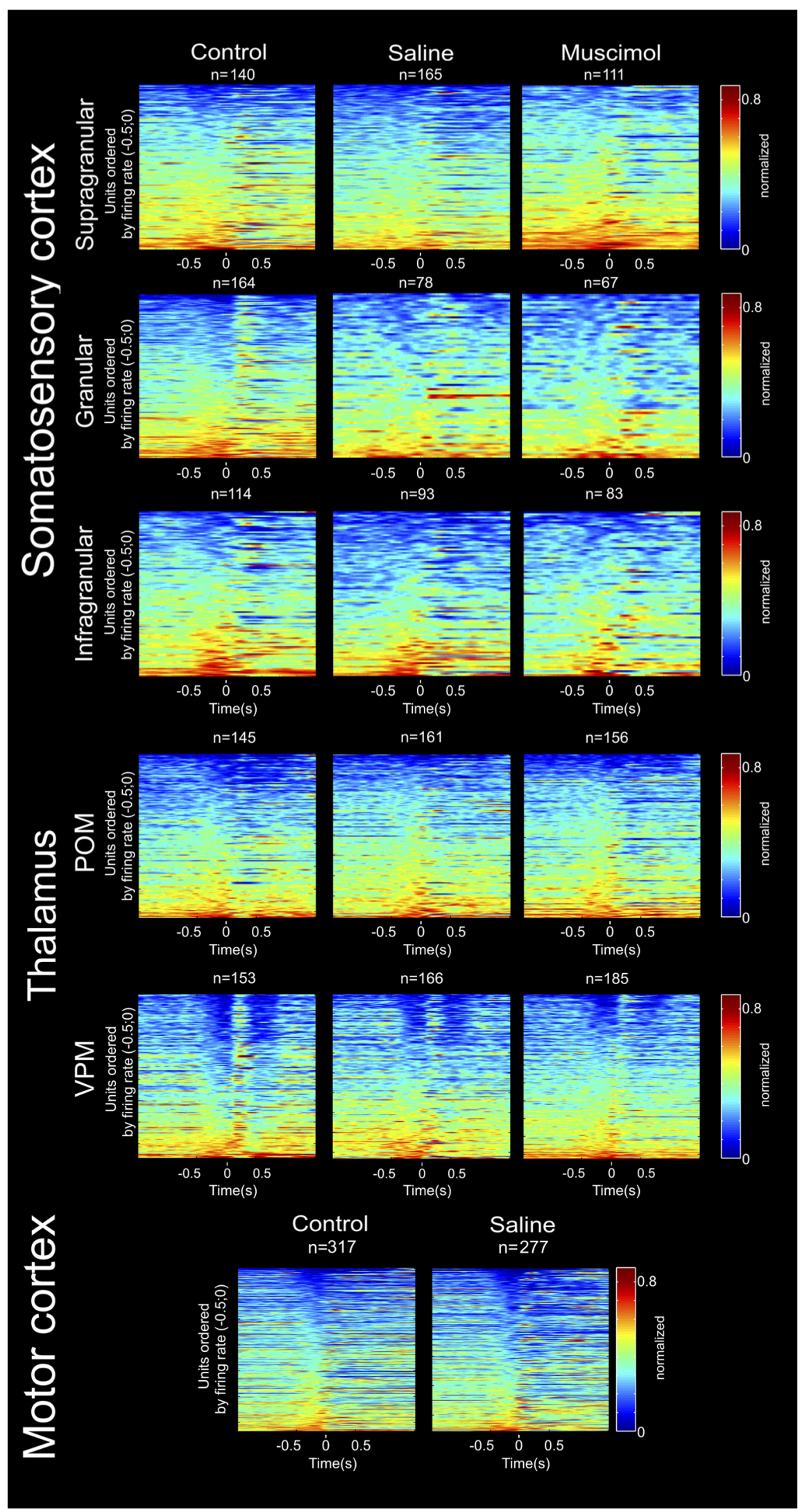

Figure 3. Neural ensemble activity across multiple thalamocortical loops during active tactile discrimination. Each row in each panel represents the activity of a single unit, normalized to its maximum firing rate, during a session. Each panel represents the activity of a different structure (from top to bottom: S1 supragranular layers, S1 granular layer, S1 infragranular layers, POM, VPM, and M1). Each column represents a different experimental condition (from left to right: control, saline injection in M1, and muscimol injection in M1). Each of the different colors represents a variation in the firing rate with red indicating excitation and chamber's door, whisker contact with the aperture edges (immediately after beam break), and whisker contacts with the center nose poke. These accounted for the sensory-evoked responses of $81.73 \%$ ( 528 of 736 single units or multiunits) of the TG neurons recorded. The firing patterns of all TG neurons recorded in this study (31 single units and 705 multiunits) are depicted in Figure $6 B$. Neurons in the TG exhibited clear sensory-evoked responses just after time 0 (beam break), indicating that these first-order cells fired maximally immediately after the whiskers contacted the aperture edges. Interestingly, a large percentage $(68.42 \%, 442$ of 736$)$ of TG neurons also exhibited significant decreases of activity as rats ran through the corridor that separated the door from the beam break. These modulations can be explained somewhat by the fact that during the period used to collect baseline firing data (before the door opened), the rats' whiskers often made contact with the surface of the closed doors.

Using the same PSTH analysis used for examining the cortical and thalamic data, we observed that a meager $5.02 \%$ of the neurons ( 37 of 736 of the units/multiunits) showed increased activations around the $250 \mathrm{~ms}$ before beam break. Careful analysis of the trials in which these 37 TG neurons fired revealed that such sensory-evoked responses were due to late whisker contacts with the chamber doors. In another words, these sensory-evoked responses did not occur during the anticipatory period. Restricting the time window to -0.2 to $-0.05 \mathrm{~s}$ to omit such occasional late whisker contacts with the doors reduced the number of excitatory responses in the anticipatory period to $1.902 \%$ (14 of 736 of the multiunits). Comparison of the activity occurring in the interval between -0.3 and $0 \mathrm{~s}$ (Fig. $6 C)$ further showed that the TG presented a period of increased activity coinciding

deep blue indicating inhibition. Time 0 corresponds to the discrimination bar beam break. Units were ordered by the maximum firing rate in -0.5 to $0 \mathrm{~s}$. In all panels, the bottom rows of cells presented increased firing rates immediately before the whiskers contacted with the discriminanda (time 0 ). These patterns were region specific, and the patterns of increased activity in a group of units were accompanied by a symmetrical pattern of decreased activity in another group, both in the same and in different regions. For example, notice that neurons in VPM and S1 granular layer presented marked anticipatory inhibitory firing, which was immediately followed by a firing increase after the whiskers contacted bars. A symmetrical pattern of increased anticipatory activity followed by inhibition is present in $\mathrm{S} 1$ infragranular layers and POM. 


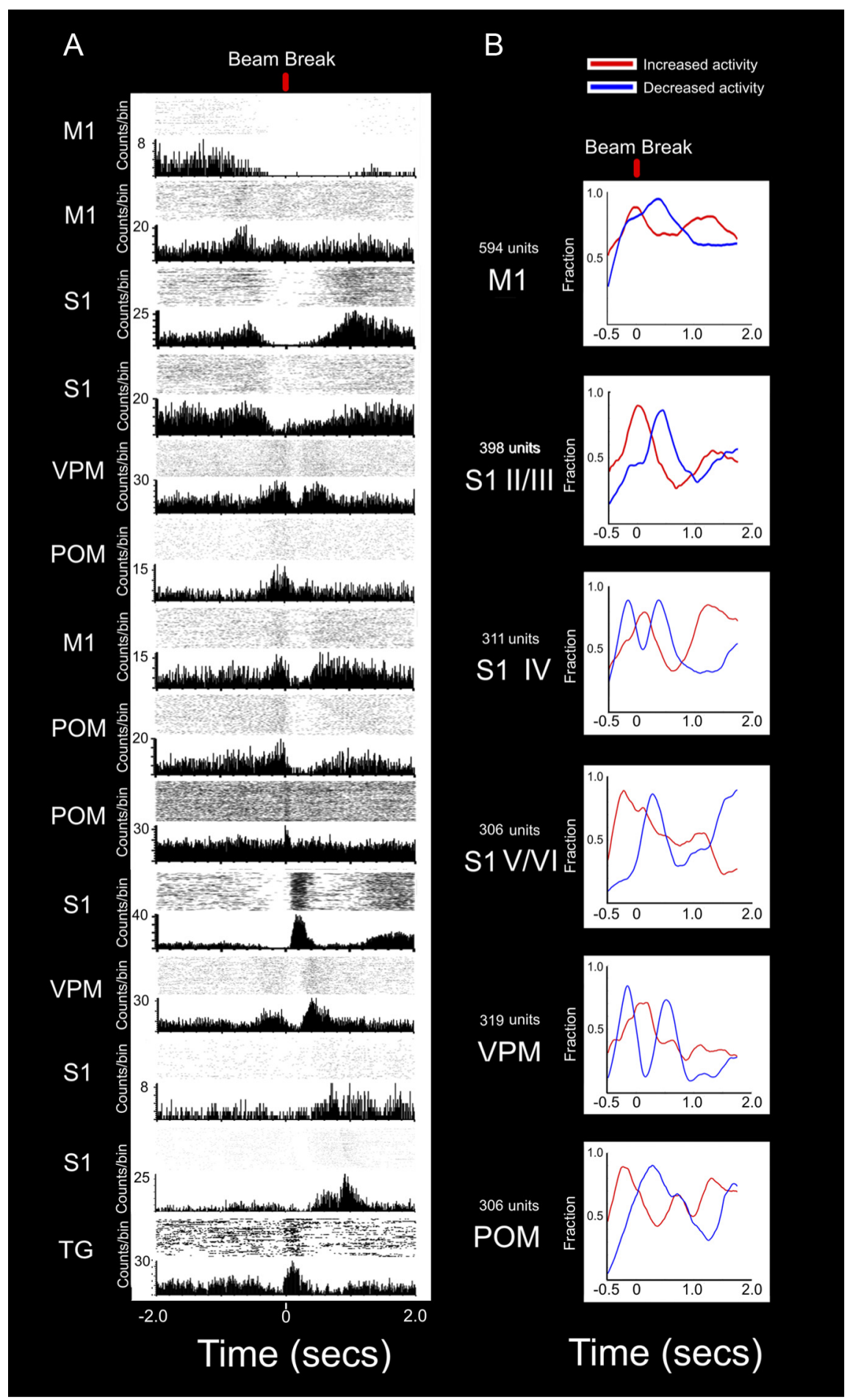

Figure 4. Ranking of neuronal ensembles reveals extensive anticipatory firing activity in M1, S1, VPM, and POM. A, PSTHs of all areas studied showing different periods of increased or decreased activity spanning across the whole length of a trial. Time 0 corresponds to the discrimination bar beam break. Cells presented are not from the same animal. The top cell was recorded in M1 and presented a period of increased activity only before the trial started. As soon as the door opened, this cell decreased its activity. The onset of this decreased activity matched the beginning of firing increases observed in other M1 and in S1 neurons (second to fourth rows). This suggests an initial role for $\mathrm{M} 1$ at the preparatory stages of a trial, followed by a second class of cells both in $\mathrm{M} 1$ and $\mathrm{S} 1$ related to early anticipatory activity as the door opens (approximately $-0.5 \mathrm{~s}$ ). As the animal moved from the door to the discrimination bars, anticipatory cells in VPM, POM, and M1 (fifth through eighth rows) exhibited a sharp increase in activity that ended as the whiskers contacted the bars (time 0 ). Although not shown in this figure, cells with anticipatory increases of firing rate were present in all structures recorded. As this group of anticipatory cells decreased its activity, a different group of cells in POM, M1 and S1 (9th through 11th rows) presented an increase in activity. This period coincides with the whiskers sampling the discrimination bars. Also, as the whiskers touch the center nose poke and the rat chooses one of the reward ports (12th and 13th rows) firing increases were observed both in VPM and S1. Notice that after the whiskers had sampled the discrimination bars, increases with the animal's whiskers contacting door. Again, this epoch did not match the period of increased anticipatory activity observed in VPM and S1. This point is highlighted even further when individual PSTHs of simultaneously recorded TG, VPM, and S1 neurons in three different rats are plotted together (Fig. 6D). This plot shows that after TG neurons respond to the whiskers contacting the doors, their firing rate tends to decrease rapidly to almost zero. Thus, S1 and VPM increases in anticipatory firing tend to occur precisely during the period in which TG neurons are virtually quiet. However, when the animal's whiskers touched the aperture edges, immediately after the beam break (Fig. 6D, bottom, BB, PSTHs), neurons in all three regions (TG, VPM, and S1) produced vigorous firing increases.

In conclusion, our control data, involving the largest sample to date of TG neurons recorded in behaving rats, clearly

\section{$\leftarrow$}

of activity started to appear again in some of the upper rows neurons, suggesting that their activity was temporarily inhibited during tactile discrimination. On the bottom row, the activity of a typical TG neuron is presented. Between the door and the discrimination bars ( $\sim 250 \mathrm{~ms})$, there is almost no activity in this neuron, indicating that no whisker contacts or movements were made. A clear increase in $\mathrm{TG}$ activity is observed as the whiskers make contact with the tactile discriminanda. Overall, the combined PSTHs presented here show that active tactile discrimination results from complex interactions where all regions are likely to have a significant contribution at every point in time, and not just during a specific epoch (e.g., motor or tactile periods). $\boldsymbol{B}$, Each line represents the fraction of neuronal firing modulations that showed increased or decreased activity at each moment during the trial. Neuronal activity from all recorded structures is aligned to show how different areas present different patterns of increased and decreased activity during a trial. The top panel shows that at the beginning of the trial, M1 starts with a marked period of increased activity (red trace), which contains the largest and earliest fraction of significant responses in all regions. These significant increases of activity end at the moment the whiskers contact the discrimination bars, after which a period of decreased in firing activity follows. A similar pattern of increased anticipatory activity, followed by a marked decrease during the discrimination phase, was also present in $\mathrm{S} 1$ layers V/VI and in POM (fourth and sixth rows, respectively). In VPM and S1 layer IV, a very distinct sequence of decrease-increase- decrease in firing activity was observed. The initial decrease in firing coincides with the period of increased anticipatory activity observed in M1, S1 layers V/VI, and POM, suggesting that the motor cortex could be gating these $\mathrm{S} 1$ and VPM neurons. Conversely, the period of maximum firing increase is present immediately after the whiskers sample the discrimination bars, marking the arrival of peripheral tactile related information. Last, layers II/III of S1 present a firing increase centered at the moment of the discrimination bar beam break, which is followed by a marked decrease of activity. This unique pattern of activity suggests that layers II/III could be fundamental for the integration of anticipatory and tactile information during the bars sampling period. 


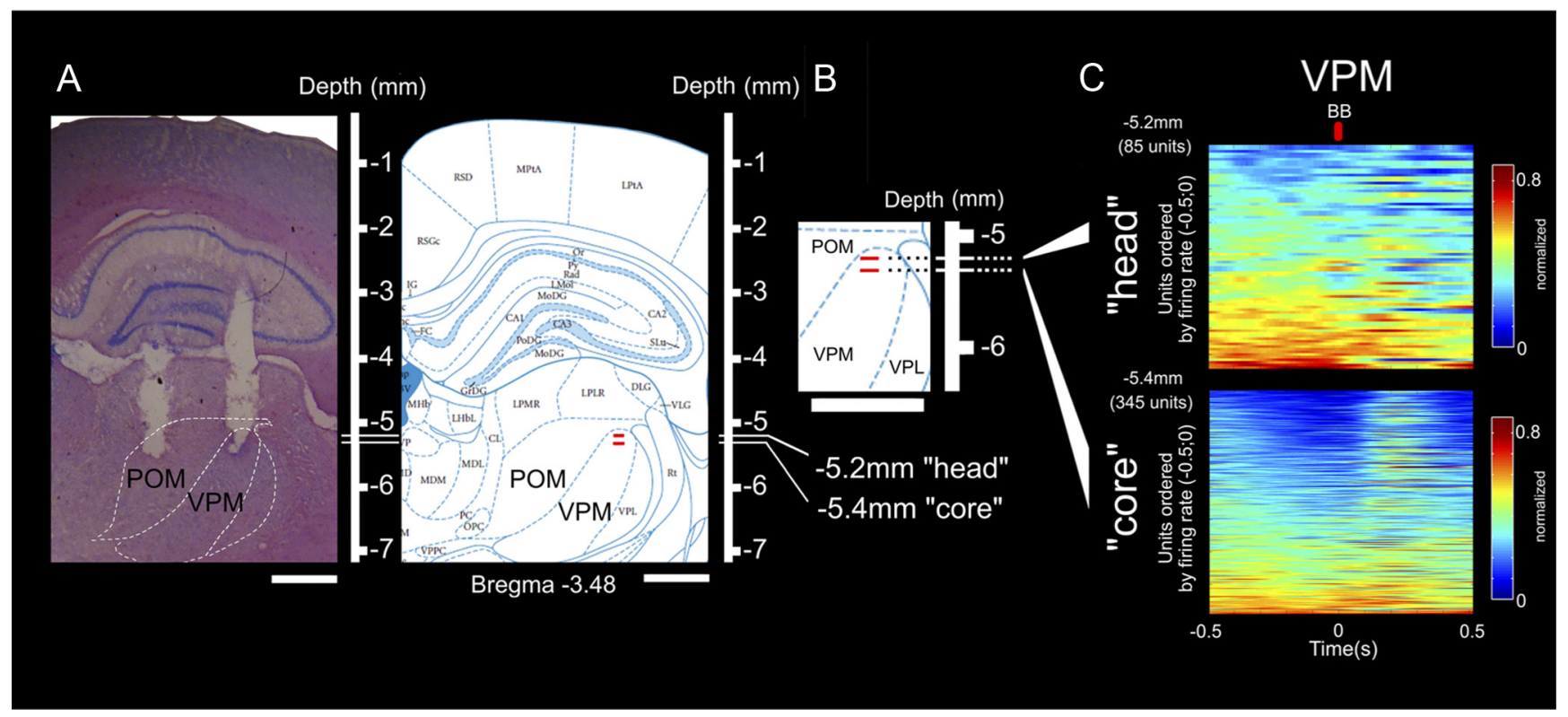

Figure 5. Both head and core of barreloids in VPM present anticipatory neural activity. $\boldsymbol{A}$, Histological verification (left) and comparison with standard diagrams (right) (Paxinos and Watson, 1998) of microelectrode placement in the POM and VPM. The white lines in the rulers and the two red markings indicate the depths at which neural activity corresponding to the head ( $-5.2 \mathrm{~mm}$ ) and core $(-5.4 \mathrm{~mm})$ regions of the VPM was recorded. $\boldsymbol{B}$, Magnification of standard diagram showing the depths used to define head and core of the barreloids. $\boldsymbol{C}$, Top and bottom show the neural ensemble activity recorded from depths corresponding to head and core of the barreloids. Each row in each panel represents the activity of a single unit during a session normalized to its maximum firing rate. Each of the different colors represents a significant variation in the firing rate, with red indicating excitation, and deep blue indicating inhibition. Time 0 corresponds to the discrimination bars $B B$. Units were ordered by the maximum firing rate in -0.5 to $0 \mathrm{~s}$. In both the head and core of the VPM, the bottom rows of neurons exhibited increased anticipatory firing rates immediately before the whiskers contacted with the discriminanda (time 0 ). This pattern of anticipatory increased activity was more pronounced in the head of the barreloids than in the core. In the core of the VPM, the period of anticipatory activity was mainly characterized by a strong inhibition before and after the whiskers sampled the aperture bars. During the discrimination period, a marked increase in firing activity was present in the core of the VPM. A similar increase was not as evident in the head of the VPM. These results show that anticipatory firing could be found at all depths studied in the VPM, but that each of the two different compartments of this thalamic nucleus displayed a very specific pattern of firing modulation. In the head of the VPM, the pattern of activation was closer to the one described for M1, POM, and S1 infragranular layers, while in the core of the VPM, the pattern was closer to the one observed in granular layer of S1. VPL, Ventral posterior lateral thalamic nucleus.

indicates that the anticipatory activity observed in S1, VPM, and POM during the period the animal crosses the corridor that separates the door from the aperture edge cannot be explained by peripheral activation of first-order TG neurons.

Yet, since well-trained animals would typically protract their whiskers to perform this task, one could argue that in some trials whisker contacts could have occurred slightly earlier than the beam break. To additionally test whether primary afferent neuron activation could occur during the time required for the animals to cross the corridor that separated the door and the aperture, we reanalyzed video recordings presented previously (Wiest et al., 2010) and calculated the difference between whisker contacts and beam break in 24 sessions. The video analysis directly showed that the rat's whiskers had no contact with any surface before the moment they touched the aperture edges. Also, the distribution of the timing, within a trial, of whisker contacts with the bar showed that typical whisker contacts (43.0\% of the trials) occurred at Frame 0 (the video frame of contact is the same as the frame of beam break) or at $-20 \mathrm{~ms}$ ( $44.2 \%$ of the trials; the video frame of whisker contact immediately precedes the frame of the beam break). Since the onset of neuronal anticipatory firing activity in M1, S1, VPM, and POM typically starts at -250 $\mathrm{ms}$, even if the onset of TG activity was further corrected for the possibility of whisker contacts at $-40 \mathrm{~ms}$ (which would include $96.8 \%$ of the trials analyzed), we would still observe clear peaks of anticipatory neuronal activity in M1, S1, VPM, and POM that cannot be explained at all by early whisker contacts.

Together, these two control experiments, as well as extensive data already published (Krupa et al., 2001, 2004; Wiest et al., 2010) rule out the hypothesis that increased S1, VPM, and POM anticipatory activity before the beam break is in any way related to early whisker mechanical stimulation by spurious whisker contacts with the chamber walls or floor.

\section{Anticipatory firing in the $S 1$ and thalamic nuclei are not due} to reafference of whisking signals

Having excluded the possibility of early whisker contacts, we tested the possibility that increased neuronal activity before contact with the tactile discriminanda could be due to some other form of whisker movements that led to sensory reafference. Using video recordings, we repeatedly observed that no whisking of any sort occurred as rats performed this tactile discrimination task (Krupa et al., 2001, 2004; Wiest et al., 2010). Instead, well-trained animals tend to spread their whiskers, which seems to improve their tactile perception of approaching objects (Krupa et al., 2001, 2004; Wiest et al., 2010). We refer to this type of whisker positioning as the object-detection mode. This behavior, which is present in very well-trained rats moving at a high speed, was described only recently (Arkley et al., 2011). In our experiments, animals also tended to perform the task at a high locomotion speed as well, and sample the tactile discriminanda for a very small amount of time (Wiest et al., 2010). Yet, to test for the possibility that the anticipatory increases in S1 and thalamic neuronal activity could be related to reafferent peripheral inputs produced by some other type of whisker positioning, we further conducted recordings in three rats (two implanted in the VPM and one implanted in both VPM and POM) with bilateral facial nerve lesions. In the same animals, we also recorded EMGs from the whisker pad as a control for facial musculature activation. By simultaneously recording EMG activity and neuronal activity 


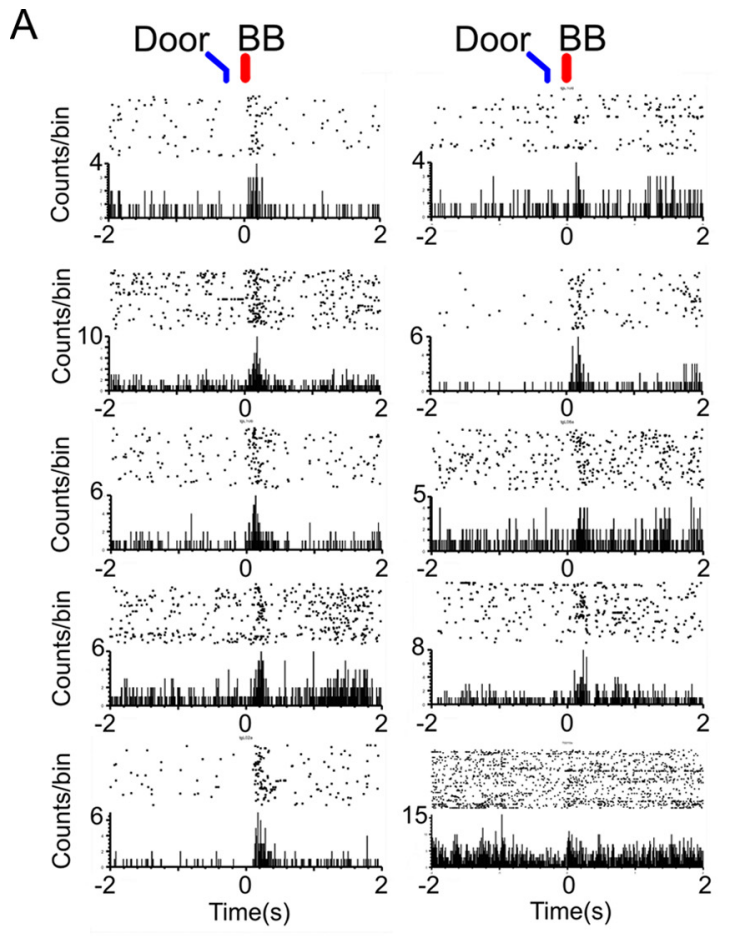

B
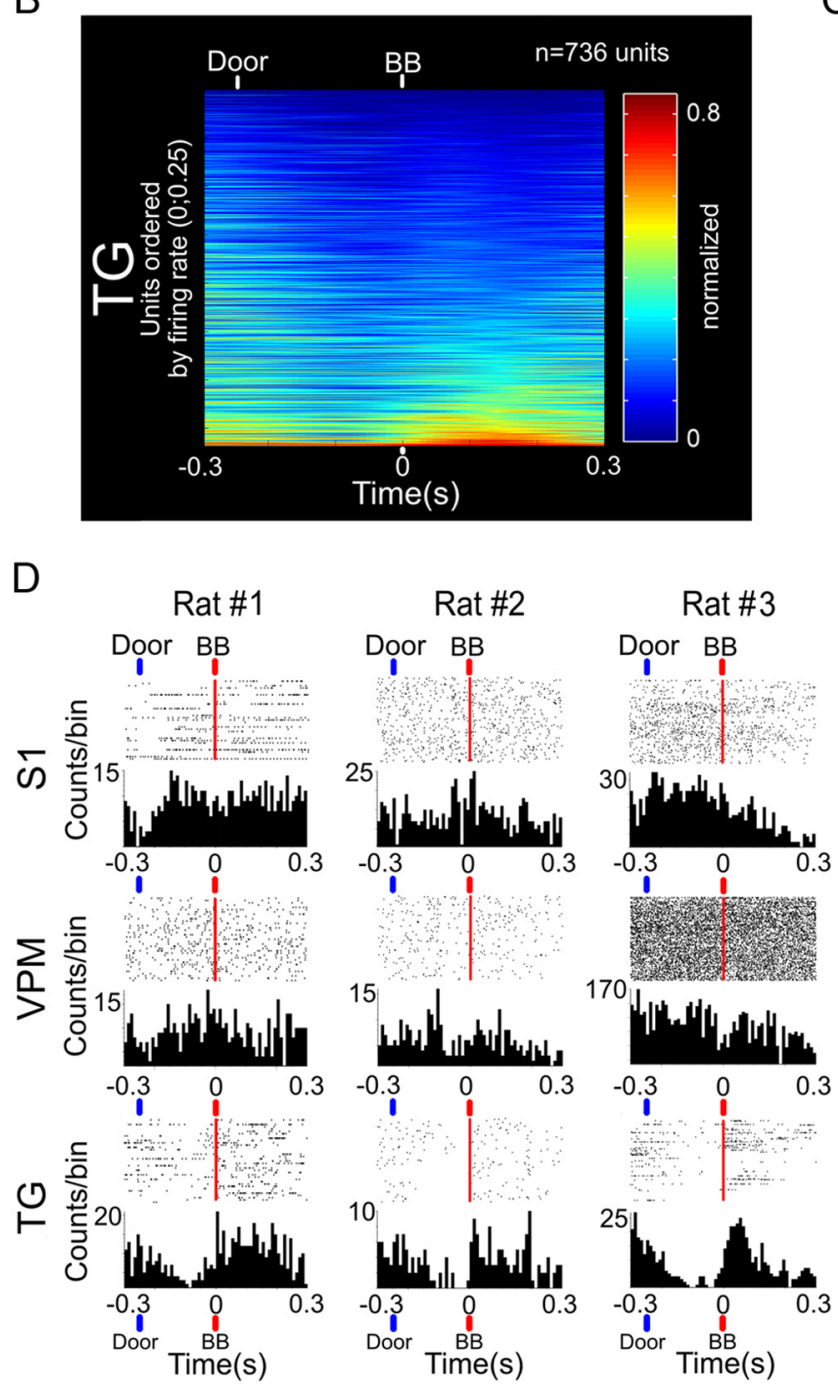

Door $\mathrm{i}^{\mathrm{BB}}$ Door $\mathrm{BB}$
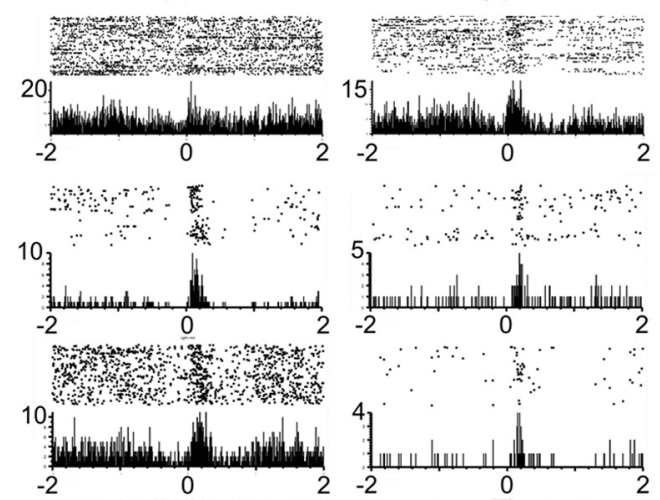

mond
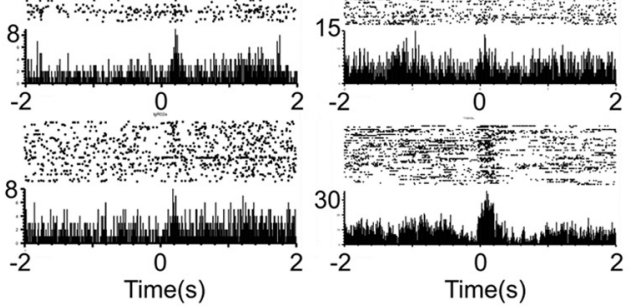

C

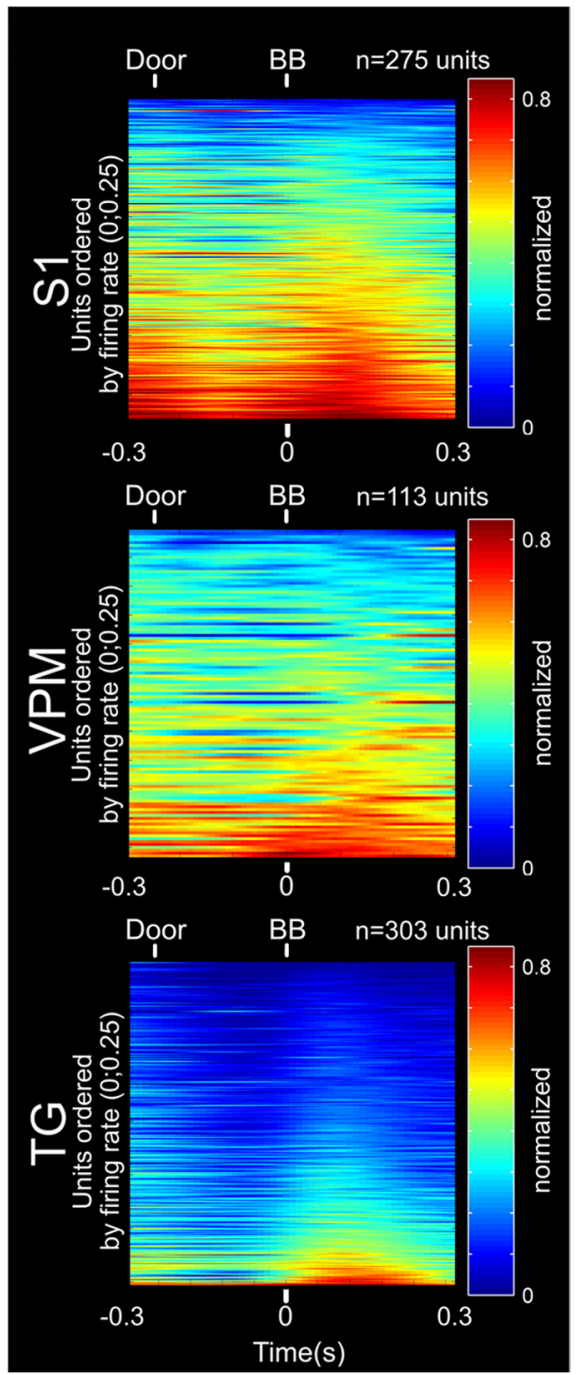



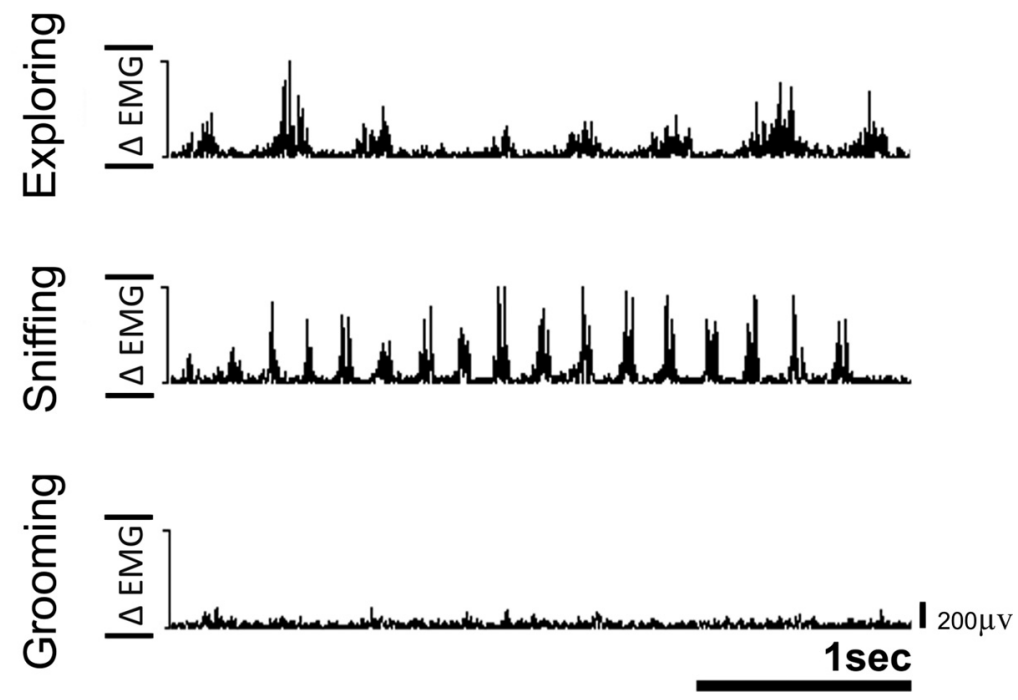

Figure 7. Examples of rectified EMG activity recorded from a rat with bilateral facial nerve lesion in an open field during three typical behaviors. After bilateral facial nerve lesion, rats learned that sniffing or chewing allowed them to make small whisker movements. Analysis of EMG peak activity allowed detection of such movements. The frequencies of the EMG events correspond to the following behaviors in open field: exploring $(\sim 4 \mathrm{~Hz})$, sniffing/twitching $(7-12 \mathrm{~Hz})$, and grooming. None of this EMG activity was present during anticipatory period (see Results).

from thalamic nuclei, we were able to measure directly whether anticipatory neuronal activity was related to whisking. Overall, we found that bilateral facial nerve lesions prevented the animals from positioning their whiskers in the object-detection mode as well as from making large exploratory movements. EMG recordings allowed detection of small facial muscle contractions or artifacts associated with the possibility of wall contacts.

After recovery from surgery, these animals quickly learned that chewing or sniffing allowed them to make small whisker movements, although they could no longer make the large exploratory whisking movements or position their whiskers in the object-detection mode. These small whisker movements were

$\leftarrow$

Figure 6. Trigeminal ganglion activity is phase locked to the tactile stimulus contact and does not appear during the anticipatory firing period. $A$, Example of trigeminal ganglion PSTHs during active aperture discrimination. The panels show PSTHs (10 ms bins), with respect to the aperture bar beam break (time $=0$ ), of trigeminal ganglion single unit and multiunits recorded during the tactile discrimination task. Between the door (blue) and the beam break (red), there is an overall reduction or absence of activity in the TG neurons, indicating that no whisker movements or contacts are present during this period. $\boldsymbol{B}$, Each row in the panel represents the activity of a single unit or multiunit, normalized to its maximum firing rate, during a session. Each of the different colors represents a variation in the firing rate, with red indicating excitation, and deep blue indicating inhibition. Time 0 corresponds to the discrimination bar beam break. Units were ordered by the maximum firing rate in 0 to $0.25 \mathrm{~s}$. A total of 736 units and multiunits recorded from five rats in 28 sessions are presented. The periods of increased activity reflect whisker contacts with the door, discrimination bars and center nose poke. A marked decrease in TG firing rate is observed in the period -0.25 to $0 \mathrm{~s}$ between the door and the discrimination bars. $C$, The activity levels presented for TG, VPM, and S1 were recorded simultaneously ( $n=3$ rats). Between the door and the discrimination bars (from -250 to $0 \mathrm{~ms}$ ), both VPM and S1 presented a significant group of cells with increased activity. In the trigeminal ganglion, this activity was almost absent (for details, see Results). Comparison of the fraction of significant increased responses (red lines in right column) showed that both VPM and S1 presented anticipatory increases in activity that did not match the TG increase. However, immediately after the beam break, all three regions presented a simultaneous peak of increased activity. $\boldsymbol{D}$, Each column demonstrates PSTHs of neurons recorded from the same session in three different animals. While the TG presented a marked reduction of activity in the $250 \mathrm{~ms}$ before the BB, sustained or phasic increases in the VPM and $\mathrm{S1}$ could still be observed. The presence of $\mathrm{S} 1$ and VPM modulations in the absence of TG activity indicate that the origin of anticipatory activity cannot be due to the activation of primary whisker afferents from the TG. easily detected by the EMG activity. Figure 7 shows the EMG activity of one of these animals in an open field. Different frequencies of EMG events were found for exploring, sniffing, and grooming in an open field.

We then recorded neural and EMG activity while the animals performed the tactile discrimination task ( $N=4$ sessions). We found that, on average, the animals displayed detectable EMG activity during the anticipatory period in only $7.64 \pm 3.4 \%$ of the trials. This value is below the $\sim 15 \%$ reported previously by us (Wiest et al., 2010), possibly due to the bilateral facial nerve lesions. After removing the trials where EMG events were present, analysis of neural activity showed that anticipatory modulations were present in $40.0 \%$ (26 of 65 ) of the thalamic (VPM and POM) units and multiunits recorded, a value that is virtually identical to those found in our control experiments. In Figure $8 A$ we show examples of several trials with clear anticipatory thalamic activity in the absence of any EMG events. In all the trials shown in this figure, thalamic anticipatory firing began $>100 \mathrm{~ms}$ before the beam break occurred, excluding the possibility of early whisker contacts (which as demonstrated above can be ruled out with an extremely conservative measure of up to $40 \mathrm{~ms}$ ). Trials 2 and 4 in Figure $8 \mathrm{~A}$ also show that EMG activity (red triangles) did not necessarily evoke any increases in neuronal firing either during the anticipatory period or after the beam break.

Next, in all rats, EMG activity was cross correlated with the beam break and with the EMG events (Fig. $8 B$ ). While EMG events were surrounded by peaks of EMG activity reflecting the frequencies of behaviors observed (note the repeated peaks at $\sim 6$ $\mathrm{Hz}$ ), no clear peak of EMG activity occurs before the beam break. Last, comparison of neuronal activity centered at the beam break or at the EMG events (Fig. 8C) further suggests that the peaks of activity related to anticipatory activity or related to EMG events occur in fundamentally different classes of thalamic neurons. These results demonstrate that the type of anticipatory neuronal activity observed in $\mathrm{S} 1$ and thalamic nuclei cannot be caused at all by sensory reafference related to whisker positioning or whisker movements.

Having ruled out the possibility that anticipatory neuronal activity was originated by early whisker contacts or early whisker movements, we further analyzed whether this anticipatory activity could result from a top-down signal originating from the primary motor cortex.

\section{Anticipatory firing modulations in lemniscal and paralemniscal pathways can be explained by two principal components}

The multitude of increased and decreased anticipatory neuronal firing modulations found in all thalamic and cortical regions studied here suggests that the TCLs continuously integrate information from both ascending and descending pathways, originating at subcortical and cortical levels. To determine whether the similar patterns of neuronal activity observed at cortical and thalamic levels were the result of largely independent modulations or, alternatively, they reflected wide interregional and correlated 

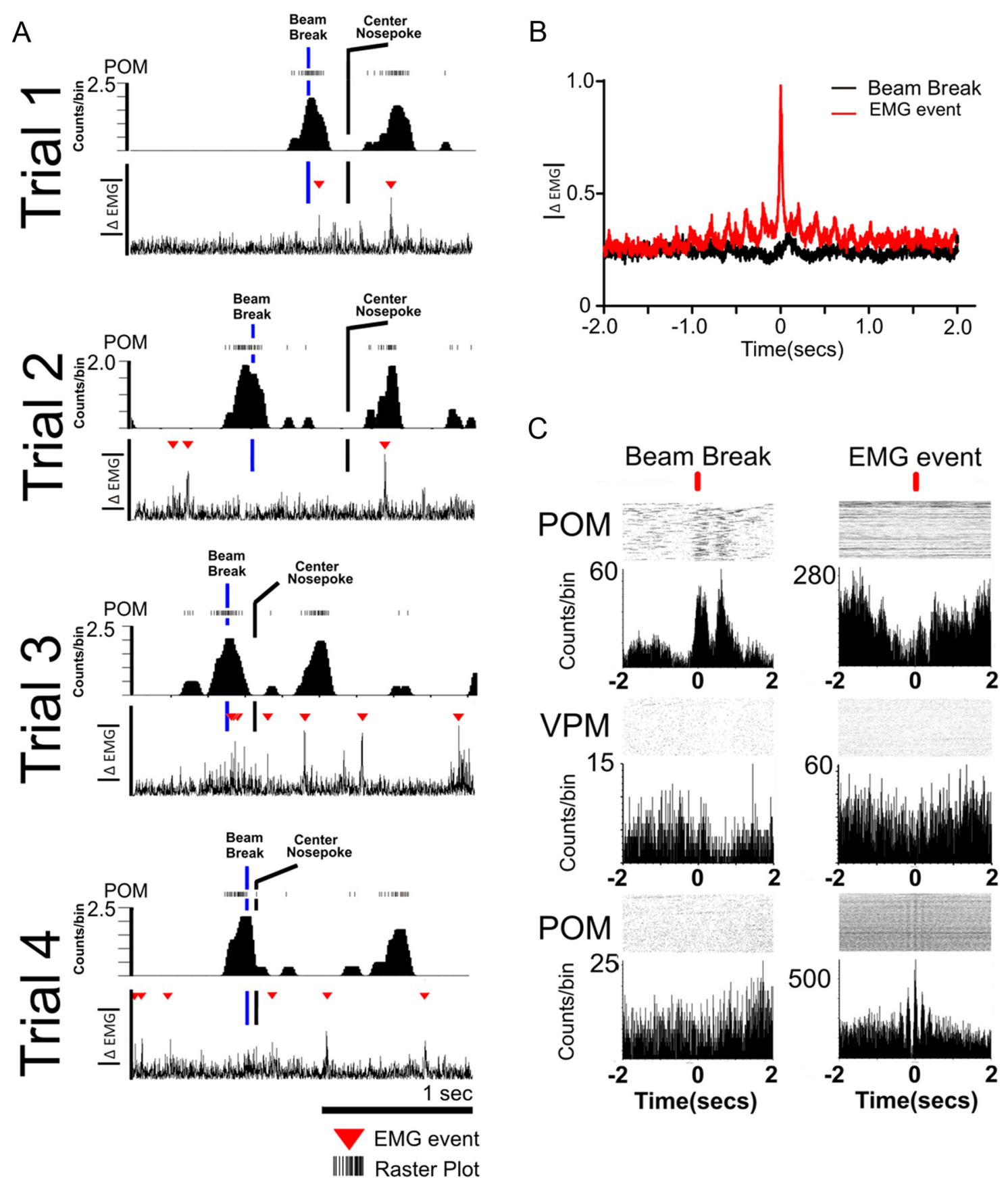

Figure 8. Anticipatory activity is independent of EMG events. $A$, Examples of four different trials in which increased anticipatory neuronal activity in a POM neuron did not match small EMG events. The top of each trial shows the raster plot for the POM neuron. Below the raster, a PSTH represents the number of counts per bin (smoothed with a Gaussian window of $30 \mathrm{~ms}$ ) of the same cell. On the bottom, the rectified EMG activity is displayed together with red triangles showing EMG events (defined as 3 SDs above the overall activity of the session). Since this rat underwent bilateral facial nerve sections, the anticipatory firing increases shown by this POM neuron cannot be explained by the typical whisker positioning used by animals to perform this task. After removing the trials where EMG events were present, the anticipatory activity of this cell was still highly significant. The same was true for $80 \%$ of POM and VPM neurons displaying anticipatory firing before the beam crossing . $B$, Average normalized (to a maximum of 1) EMG activity recorded from the three rats around the beam break and around the EMG events. No significant increase in EMG activity was observed before the beam break, indicating that EMG events were mostly absent during the period of anticipatory firing observed in the S1, VPM, and POM during execution of this task. C, Anticipatory activity and EMG event-related activity occur in different groups of cells. Activity of three neurons relative to beam break (left) and to EMG events is shown. The top neuron presented significant increased anticipatory activity for the beam break, but not for the EMG events, suggesting that anticipatory activity was independent of the EMG signal. The middle neuron showed a small increase of activity immediately after the beam break, but no clear changes around the EMG events. In contrast, notice that the bottom neuron is phase locked to the EMG events, but not to the tactile discrimination task. The differences found in neurons that presented EMG-related or anticipatory activity suggest that fundamentally different classes of neurons were activated around EMG events or during the anticipatory period.

modulations across the TCLs, we performed a principal component analysis on all increased and decreased firing modulations in M1, VPM, POM, and each S1 layer for the period of -0.5 to $1.0 \mathrm{~s}$. This analysis revealed that the first two principal components accounted for $71.03 \%$ of the variance of the entire data set (Table 2). When a third principal component was added, $93 \%$ of the variance of the firing patterns observed across multiple cortical and thalamic structures was explained. This result clearly sup- 
Table 2. Principal component analysis of increased and decreased activity

\begin{tabular}{|c|c|c|c|c|c|c|c|c|}
\hline & Cumulative variance (\%) & pc variance & & Response & $p c 1$ & $\mathrm{pc2}$ & $p c 3$ & pc4 \\
\hline $\mathrm{pc1}$ & 44.21030281 & 0.1629294 & Supragranular & Increased & 0.192969 & 0.4848809 & -0.312331 & 0.0005789 \\
\hline pc2 & 71.03246885 & 0.0988484 & & Decreased & 0.4076718 & -0.205153 & 0.1687933 & -0.0543012 \\
\hline pc3 & 92.99443819 & 0.080937 & Granular & Increased & 0.166108 & 0.2601034 & -0.3648693 & -0.0260106 \\
\hline pc4 & 96.89447643 & 0.0143729 & & Decreased & 0.3399926 & 0.2139566 & 0.2859596 & -0.5156428 \\
\hline pc5 & 98.60621637 & 0.0063083 & Infragranular & Increased & 0.0512769 & 0.4105422 & -0.0402117 & -0.1843402 \\
\hline pc6 & 99.61462124 & 0.0037163 & & Decreased & 0.4235365 & -0.2734356 & -0.2119353 & -0.3806003 \\
\hline pc7 & 99.83804586 & 0.0008234 & M1 & Increased & 0.084304 & 0.1439821 & -0.1092586 & 0.3450707 \\
\hline pc8 & 99.94689553 & 0.0004011 & & Decreased & 0.3603517 & -0.0147926 & 0.0682632 & 0.2510888 \\
\hline $\mathrm{pc} 9$ & 99.9710496 & $8.90 \mathrm{E}-05$ & VPM & Increased & 0.1967974 & 0.2950371 & -0.2542239 & 0.0260476 \\
\hline pc10 & 99.98996768 & $6.97 \mathrm{E}-05$ & & Decreased & 0.1489934 & 0.3088844 & 0.7176482 & 0.0854539 \\
\hline pc11 & 99.99776799 & 2.87E-05 & POM & Increased & -0.1273602 & 0.3553321 & 0.1261817 & 0.2783481 \\
\hline pc12 & 100 & $8.23 \mathrm{E}-06$ & & Decreased & 0.5072061 & -0.1907687 & -0.0188202 & 0.5329833 \\
\hline
\end{tabular}

Analysis of significant increased and decreased responses across all areas studied showed that two principal components (pcs) were able to explain a large portion of the sample's variance. The first component included mostly decreased activity from all S1 layers, M1, and POM, and increased activity in VPM. The second component included increased activity in all S1 layers, VPM, and POM, and decreased activity in VPM and all S1 layers. The presence of such a large portion of variance explained solely by two components indicates that common concurrent patterns of increased and decreased activity occur at all times in the TCLs, suggesting a distributed mode of processing during active tactile discrimination.

ports the existence of highly correlated patterns of anticipatory activity across the TCLs. For example, the first component included decreased activity from all S1 layers, M1, POM, and increased activity in VPM, while the second component included increased activity in all S1 layers, VPM, and POM, and decreased activity in VPM and S1 granular layer (for positive and negative loadings in each component, see Table 2). The presence of such a high portion of variance explained with only three principal components suggests that patterns of anticipatory activity are linearly correlated across cortical and thalamic structures, and that three of these neuronal patterns are sufficient to explain most of the response variability found across the TCLs. These concurrent patterns of responses also suggest that active tactile encoding is widely distributed across the multiple TC loops of the trigeminal system and results from large-scale, temporally asynchronous interactions between the many structures that define this circuit (Ghazanfar and Nicolelis, 2001).

\section{Anticipatory activity predicts tactile performance}

The presence of both cortical and thalamic neurons exhibiting anticipatory firing activity suggests that all these brain areas are engaged during the time period that precedes whisker contact with the stimulus (Figs. 2-4). Since we have shown that facial whiskers do not move during this precontact phase (see Results, above), this finding cannot be explained by the classical feedforward model of tactile processing proposed to account for the main physiological properties of the trigeminal somatosensory system. As a typical increase or decrease in cortical and thalamic neuronal activity started around $-250 \mathrm{~ms}$ relative to the whiskers' contact with the bars and ended at the time of discrimination, this anticipatory firing was related to the period that separated the crossing of the chamber door and the facial whisker contact with the aperture edges (typically $250 \mathrm{~ms}$ ).

A linear regression analysis of the relationship between the onset of anticipatory firing in cortical and thalamic units and the percentage of correct trials in each session revealed that, both in control and saline conditions, the timing of the onset of anticipatory cortical activity in $\mathrm{M} 1$ and $\mathrm{S} 1$ was a good predictor of the animal's task performance (Fig. 9C, C1, C2). Such a correlation between onset of neuronal anticipatory responses and behavioral performance was also observed for $\operatorname{VPM}\left(F_{(1,5)}=6.941 ; p=\right.$ $\left.0.0463 ; R^{2}=0.58\right)$ and POM neurons $\left(F_{(1,14)}=18.69 ; p=\right.$ $0.0007 ; R^{2}=0.57$; Fig. $\left.9 C, C 4\right)$. Therefore, the timing of the onset of anticipatory neuronal firing activity, in both the lemniscal and paralemniscal pathways of the trigeminal system, can predict the animal's tactile performance in our tactile discrimination task: the earlier the onset of anticipatory firing, the better the animal's performance. This finding suggests that this type of neural modulation may be functionally significant for sensory-motor integration in a tactile discrimination task that does not require whisker movements.

\section{M1 inactivation affects tactile discrimination}

Intracortical injection of $500 \mathrm{ng}$ of muscimol in $500 \mathrm{nl}$ of saline induced a temporary inactivation of M1. This was confirmed by an initial reduction in neural activity, followed by a complete absence of action potentials from M1 neurons recorded by the microelectrodes surrounding the injection cannula (Krupa et al., 1999; Ghazanfar et al., 2001; Shuler et al., 2002). Our M1 inactivation was very localized and did not induce any gross motor impairment such as a reduction in the number of trials performed (Fig. 2D; control, 102.9 \pm 3.15 trials; saline, $102.0 \pm 3.66$ trials; muscimol, $103.5 \pm 4.00$ trials; one-way ANOVA, $F_{(2,118)}=$ $0.04142, p=0.9594$ ) or reduced locomotion speed (control, $22.7 \pm 1.19 \mathrm{~cm} / \mathrm{s}$; saline, $26.5 \pm 1.69 \mathrm{~cm} / \mathrm{s}$; muscimol, $23.7 \pm 1.60$ $\mathrm{cm} / \mathrm{s}$; Kruskal-Wallis statistic, $0.8736 ; p=0.6461$ ). The only behavioral impairment observed in these animals was a decrease in their ability to discriminate with their whiskers a $14 \mathrm{~mm}$ difference in width between a narrow versus a wide aperture (control, $83.2 \pm 1.01 \%$ correct trials; saline, $82.9 \pm 1.66 \%$ correct trials; muscimol, $70.3 \pm 3.50 \%$ correct trials; Kruskal-Wallis statistic, $10.21 ; p=0.0061 ;$ post hoc comparisons with Dunn's test, control vs saline, $p>0.05$, nonsignificant (n.s.); control vs muscimol, $p<0.05$; Fig. 2C). As we have demonstrated repeatedly here and previously (see above), whisker movements were not required for rats to discriminate the aperture width with their vibrissae (Krupa et al., 2004; Wiest et al., 2010).

High-resolution video analysis of the whisker angles and duration of contact of the whiskers with the stimulus showed no differences across all three different conditions (control, saline, and muscimol). Comparison of whisker angles showed an overall significant effect for face side, suggesting that animals have a natural bias toward larger angles between the right whiskers and the right whisker pad $\left(F_{(1,8)}=77.57 ; p<0.0001\right.$, n.s., for post hoc analysis in all conditions; control, left, $32.32 \pm 6.085^{\circ}$; right, $41.29 \pm 4.048^{\circ}$; $t_{(6)}=1.227, p=0.2657$; saline, left, $35.38 \pm 4.754^{\circ}$; right, $43.10 \pm$ $4.389^{\circ} ; t_{(6)}=1.194, p=0.2775$; muscimol, left, $36.30 \pm 5.502^{\circ}$, right, $\left.45.96 \pm 6.235^{\circ} ; t_{(4)}=1.161, p=0.3101\right)$. Also, no interaction $\left(F_{(2,8)}=0.7364 ; p<0.7364\right)$ or experimental condition effects $\left(F_{(2,6)}=0.08416 ; p=0.9203\right)$ were found. Comparison of the 

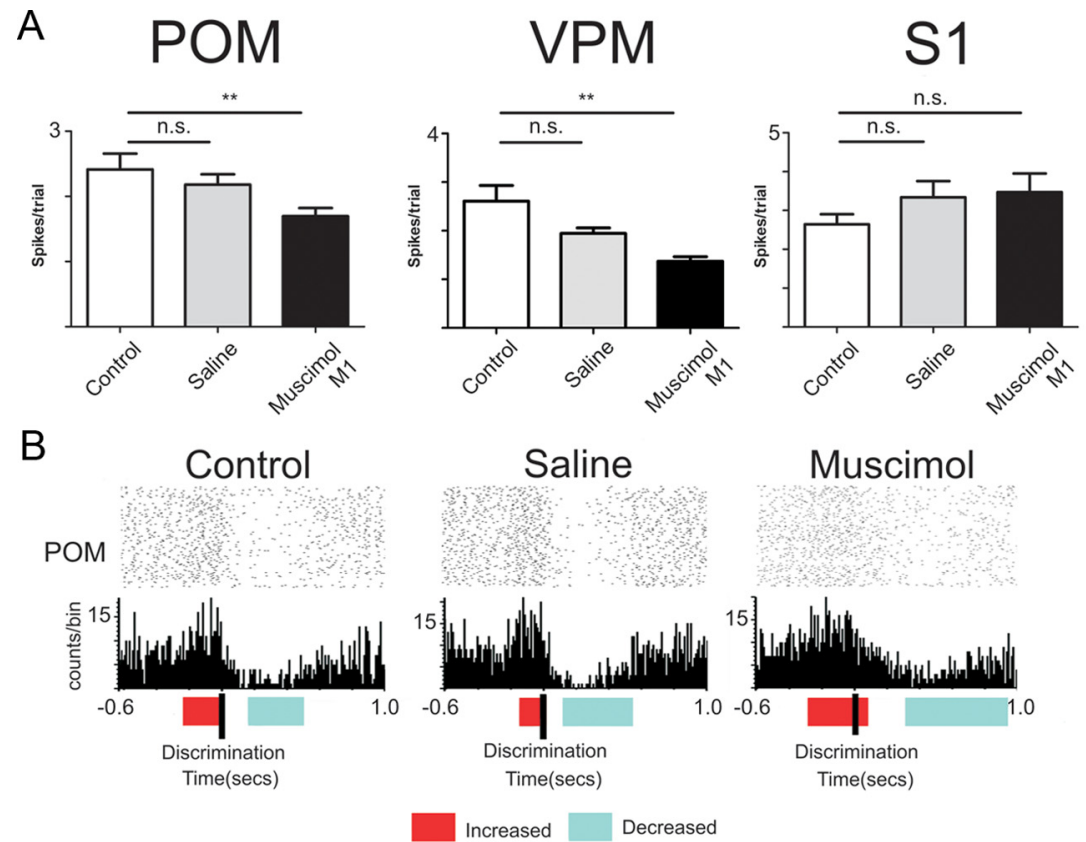

\section{Muscimol}

C

C1

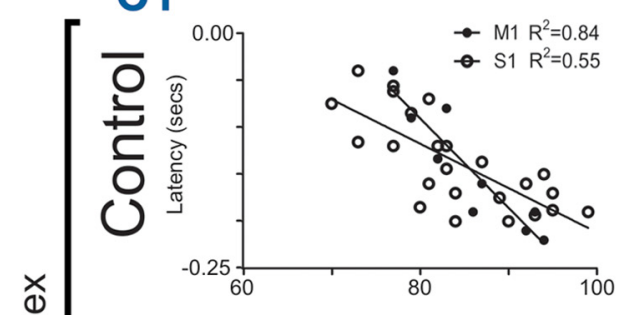

Anticipatory
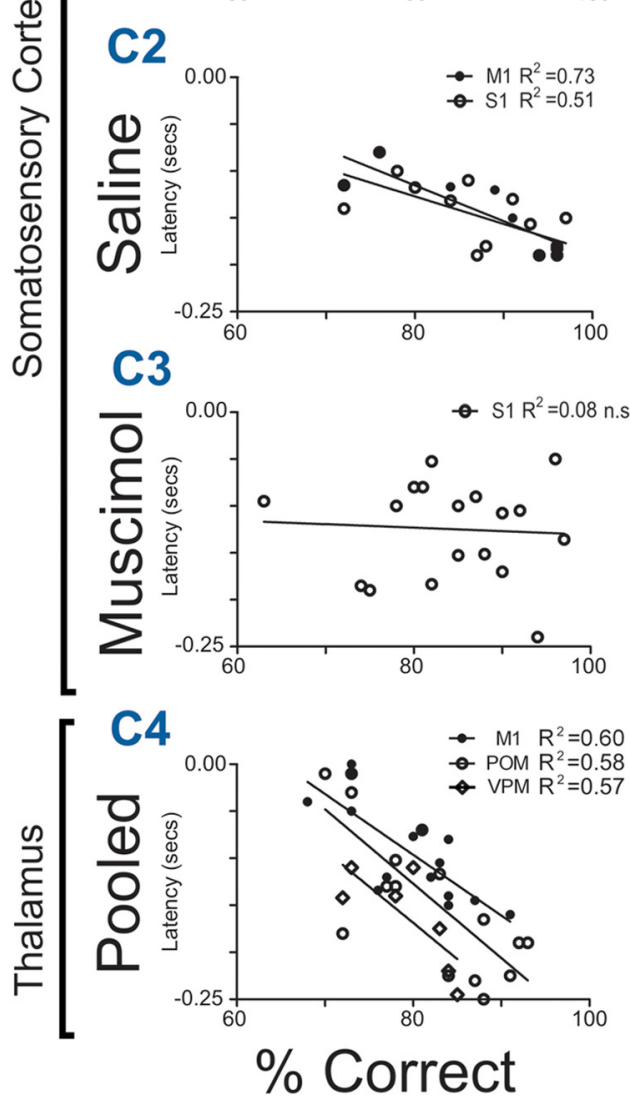
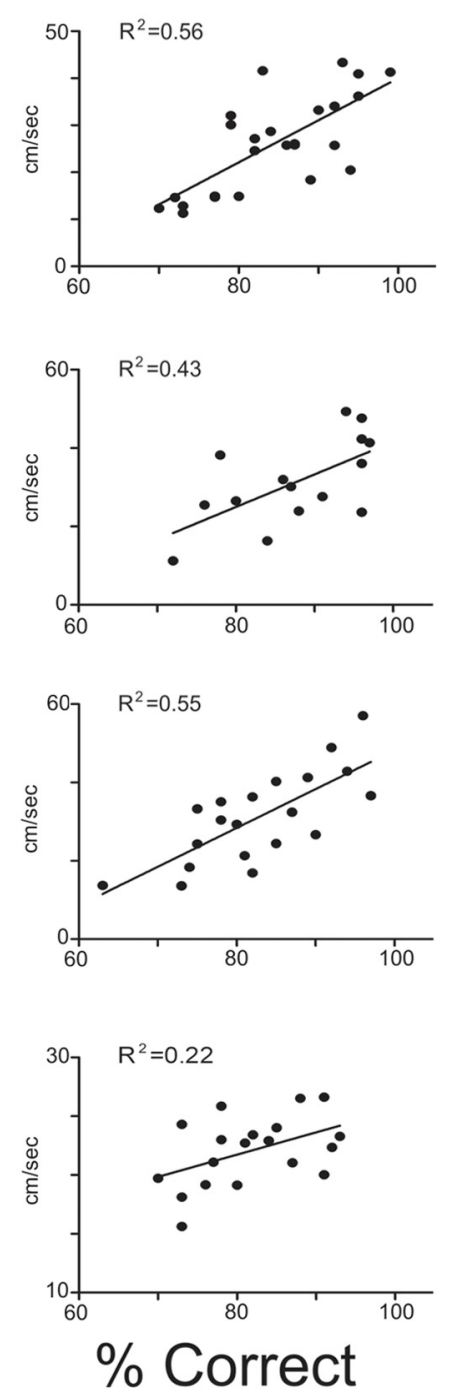

amount of time that the whiskers contacted the tactile stimulus did not differ between conditions (control, $0.245 \pm 16.74 \mathrm{~s}$; saline, $0.213 \pm 9.81 \mathrm{~s} ;$ muscimol, $0.201 \pm 12.44 \mathrm{~s}$; one-way ANOVA, $F_{(2,8)}=2.694, p=$ 0.1275).

M1 inactivation modulates anticipatory activity across the TCL

After M1 inactivation with muscimol, S1 neuronal firing modulations were widely affected. The proportion of units with increased responses in S1 rose in the anticipatory period (control, 27.9\%, 36 units; saline, 24.0\%, 29 units; muscimol, 46.2\%, 49 units; control vs saline, $\chi^{2}=0.32, \mathrm{df}=$ $1, p=0.57$; control vs muscimol, $\chi^{2}=$ 7.68 , df $=1, p=0.0056$ ), and fewer cells exhibited decreased activity in infragranular layers (control, 26.88\%, 24 units; saline, $17.72 \%, 14$ units; muscimol, $4.84 \%, 3$ units; $\chi^{2}=18.9, \mathrm{df}=2, p<$ $0.0001)$. In addition, the magnitude of increased neuronal activity was larger (control, $1.9 \pm 0.1$ spikes per trial; saline, $2.1 \pm 0.2$ spikes per trial; muscimol, $2.24 \pm 0.2$ spikes per trial; Kruskal-Wallis statistic, 6.305; $p=0.0427$; post hoc comparisons with Dunn's test, control vs saline, $p>0.05$, n.s.; control vs muscimol, $p<0.05)$. Moreover, the magnitude of

$$
\leftarrow
$$

Figure 9. Timing of anticipatory firing activity predicts animal's performance. $\boldsymbol{A}$, The inactivation of $\mathrm{M} 1$ with muscimol reduced the magnitude of anticipatory responses in VPM and POM, but not in S1. However, the proportion of cells that presented anticipatory firing increased in $\mathrm{S1}$ (for details, see Results). $\boldsymbol{B}$, Examples of an anticipatory unit recorded from POM in the same channel for 3 consecutive days, presenting similar wave shape, ISI, and average firing rate. The colored horizontal bars indicate the beginning and end of significant increases (red) and decreases (blue) in activity. This POM unit presented a similar profile of multiphasic response in all three sessions. This profile consisted of anticipatory increased activity that ended when the whiskers made contact with the tactile stimulus, followed by a period of decreased activity. After M1 inactivation, the exact timing of the anticipatory response offset was altered, suggesting that the motor cortex is involved in shaping fine details of neural anticipatory responses. C, Anticipatory activity and animal's speed as a predictor of animal's performance. The latency of the anticipatory activity onset recorded in the thalamocortical loops during control and saline sessions was a good predictor of the animal's performance in the tactile discrimination task. The earlier the onset of anticipatory activity, the better the animal's performance. Animal speed was also a very good predictor of animal's performance. The faster the animal, the better its performance (top right). After M1 inactivation, anticipatory activity no longer contained enough information to predict animal's performance. However, animal's speed remained a good predictor of tactile performance. Anticipatory onsets from VPM and POM are from control and saline sessions pooled together, since a smaller number of sessions were recorded in these conditions. Error bars indicate SEM. ${ }^{* *} p<0.01$. 
neuronal activity reduction was lowered in the infragranular S1 layers (control, $19.8 \pm 2.0$ spikes per trial; saline, $18.0 \pm 2.0$ spikes per trial; muscimol, $9.105 \pm 1.6$ spikes per trial; Kruskal-Wallis statistic, 8.523; $p=0.0141$; post hoc comparisons with Dunn's test, control vs saline, $p>0.05$, n.s.; control vs muscimol, $p<0.05)$.

M1 inactivation also led to a reduction in the magnitude of anticipatory activity both in the POM (control, $2.4 \pm 0.2$ spikes per trial; saline, $2.2 \pm 0.2$ spikes per trial; muscimol, $1.7 \pm 0.1$ spikes per trial; Kruskal-Wallis statistic, 13.57; $p=0.0011$; post hoc comparisons with Dunn's test, control vs saline, $p>0.05$, n.s.; control vs muscimol, $p<0.01$ ) and VPM (control, $2.7 \pm 0.3$ spikes per trial; saline, $1.9 \pm 0.1$ spikes per trial; muscimol, $1.3 \pm$ 0.1 spikes per trial; Kruskal-Wallis statistic, 15.05; $p<0.001$; post hoc comparisons with Dunn's test, control vs saline, $p>0.05$, n.s.; control vs muscimol, $p<0.01$ ), as shown in Figure 9A. The effects of M1 inactivation in a POM unit in three consecutive sessions are shown in Figure 9B. This cell presented similar firing rates, waveforms, ISIs, and response profiles (increased anticipatory followed by decreased discriminatory activity) in all three sessions. During control and saline conditions, the response profile shows a sharp peak of significant increased activity that begins in the anticipatory period and ends when the whiskers make contact with the discrimination bars. After M1 inactivation, the peak of activity was not as sharp as in other two conditions; the neuron's activity remained significantly high after the whiskers made contact with the bars and its period of significant reduced firing activity was longer than in the previous conditions.

To study whether the two different compartments recorded in the VPM were differentially affected by M1 inactivation, we further analyzed the magnitude of neural anticipatory activity in the head and core of the barreloids for the animals used in the inactivation experiments. A total of 77 neurons recorded from nine sessions were used for analysis of the VPM head of barreloids, while 391 neurons recorded in 33 sessions were used for the analysis of the VPM barreloids core. No significant differences were found in the anticipatory activity in the VPM head following M1 inactivation. In the core region of VPM, which sends thalamocortical projections to layer IV of S1 cortex, M1 inactivation lowered the magnitude of decreased neural anticipatory activity (control, $2.8 \pm 0.5$ spikes per trial; saline, $1.7 \pm 0.1$ spikes per trial; muscimol, $1.0 \pm 0.2$ spikes per trial; Kruskal-Wallis statistic, 18.16; $p=0.0001$; post hoc comparisons with Dunn's test, control vs saline, $p>0.05$, n.s.; control vs muscimol, $p<0.0001$ ). Also, a nonsignificant trend was found in the magnitude of increased neural anticipatory activity (control, $1.8 \pm 0.3$ spikes per trial; saline, $2.1 \pm 0.2$ spikes per trial; muscimol, $1.3 \pm 0.2$ spikes per trial; Kruskal-Wallis statistic, 9.076, $p=0.0107$; post hoc comparisons with Dunn's test, control vs saline, $p>0.05$, n.s.; control vs muscimol, $p>0.05$, n.s.). Thus, M1 inactivation induced an overall increase in significant neuronal responses in the granular and infragranular layers of S1, before and after the whiskers contacted with the aperture's edge. At the same time, the same manipulation produced a reduction in anticipatory and discriminatory activity in both the POM and the VPM.

To measure whether M1 inactivation, and the consequent changes in anticipatory $\mathrm{S} 1$ neuronal activity, affected the prediction of the animal's tactile performance, a linear regression analysis was performed between the onset of anticipatory firing in S1 units and the percentage of correct responses after M1 inactivation with muscimol. Although speed remained a good predictor of the performance in the task $\left(F_{(1,18)}=22.19 ; p=0.0002 ; R^{2}=\right.$ 0.55 ), indicating that no major motor deficits were present (con- sistent with previously unpublished observations), the onset of anticipatory units in $\mathrm{S} 1\left(F_{(1,19)}=0.3414 ; p=0.79 ; R^{2}=0.075\right.$; Fig. $9 C, \mathrm{C} 3$ ) was no longer predictive of the performance in the task. This result clearly indicates that blocking M1 activity affected spatiotemporal patterns of S1 anticipatory neural activity that predicted the animal's tactile performance.

\section{Encoding of tactile stimulus depends on anticipatory activity}

Next, we asked whether single trial alterations in anticipatory activity onset timing in S1 neurons influenced the encoding of the tactile stimulus. To achieve this goal, we first analyzed firing rate changes in neural ensemble activity before the whiskers made contact with the tactile stimulus. Specifically, for each trial, we selected the first bin presenting an ensemble firing rate that was significantly different (at $p \leq 0.05$ ) from baseline. These changes were termed neural events of interest (for details, see Materials and Methods). Note that this analysis was restricted to S1 units and that, to match previous results from our laboratory, we exceptionally used $50 \mathrm{~ms}$ bins only in this analysis.

A similar number of NEIs was found across different conditions (control, 26.23\% of trials; saline, $31.34 \%$ of trials; muscimol, $27.16 \%$ of trials; control vs saline, $\chi^{2}=2.97, \mathrm{df}=1, p=0.0849$; control vs muscimol, $\chi^{2}=1.5, \mathrm{df}=1, p=0.2207$ ), indicating that changes in neural activity occurred in a similar proportion of trials in all conditions. However, comparison of the distribution of anticipatory NEIs between the control condition and during M1 inactivation suggests that blocking M1 activity induced a major disruption in the normal timing pattern of anticipatory activity (Fig. 10). Specifically, the distribution of NEIs did not exhibit a clear peak in the interval of -400 to $-200 \mathrm{~ms}$ before contact with the stimulus (Fig. 10A). This suggests that normal M1 activity affects the precise timing of anticipatory activity in S1 neurons.

To test whether precise timing of anticipatory activity was related to tactile discrimination performance, we then compared the proportion of NEIs that were present before correct and incorrect trials in early -500 to $-200 \mathrm{~ms}$ or late anticipatory periods -200 to $0 \mathrm{~ms}$. The probability of a correct trial after an NEI was $51.6 \%$ of trials in control sessions and $45.1 \%$ of trials in saline sessions $\left(\chi^{2}=0.7921 ; p=0.1867\right)$. However, after M1 inactivation only $36 \%$ of the trials with neural anticipatory NEI were correct $\left(\chi^{2}=4.229 ; p=0.0199\right.$; Fig. $\left.10 B\right)$. These results suggest that in the absence of M1 modulation, the late onset of S1 anticipatory activity was associated with tactile discrimination deficits.

Because neurons with anticipatory activity often decreased their firing activity during contact with the tactile stimulus (Figs. $2 A, F, 9 B)$, we then tested whether anticipatory NEIs in -500 to $-200 \mathrm{~ms}$ or late anticipatory -200 to $0 \mathrm{~ms}$ periods were associated with different ensemble firing rates during the tactile encoding period 0 to $300 \mathrm{~ms}$. Comparison of the variation between the S1 ensemble firing rate before and after discrimination showed that early anticipatory NEIs were associated with larger decreases in firing rates during the tactile discrimination period in control (control early, $-0.05924 \pm 0.0059$ spikes per trial; late, $-0.03223 \pm 0.0093$ spikes per trial; Mann-Whitney $=9651, p=$ 0.0368 ) and saline conditions (saline early, $-0.03774 \pm 0.0063$ spikes per trial; late, $-0.01483 \pm 0.0077$ spikes per trial; MannWhitney $=7255, p=0.0276)$, but not after M1 inactivation with muscimol (muscimol early, $-0.06672 \pm 0.0059$ spikes per trial; late, $-0.05190 \pm 0.0098$ spikes per trial; Mann-Whitney $=6247$, $p=0.4055$, n.s.). This finding suggests that in the absence of M1 modulation, the time onset of anticipatory activity was delayed, possibly affecting the encoding of the tactile stimulus by S1 neuronal ensembles. 

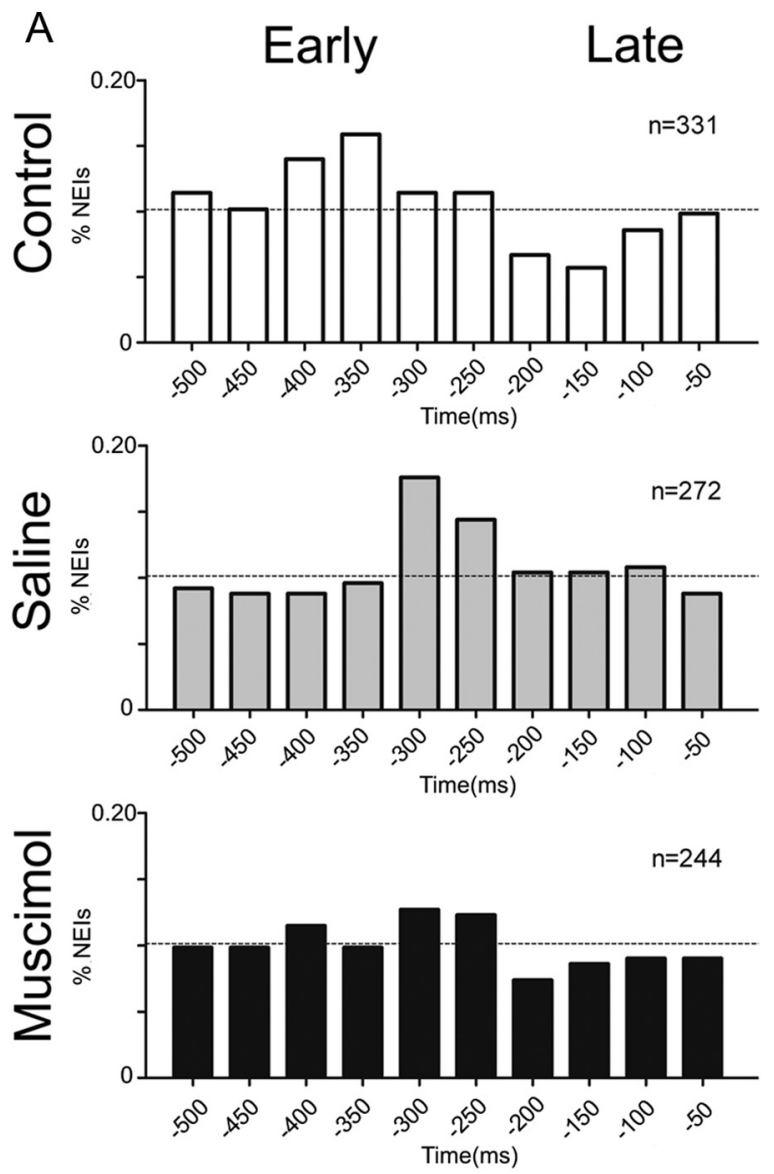

B

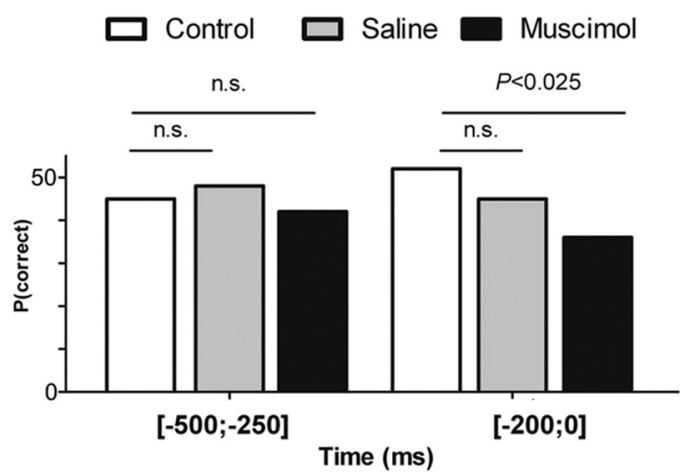

Figure 10. Trial-by-trial ensemble analysis of anticipatory neural activity. An NEl was defined as the first significant ( $p \leq 0.05$ ) neural ensemble firing modulation in the anticipatory period -500 to $-50 \mathrm{~ms}$ relative to the trial baseline period. $A$, The distribution of NEls in the anticipatory period of control and saline ensembles was concentrated around the period -400 to $-200 \mathrm{~ms}$. After M1 inactivation the distribution of anticipatory NEls was closer to a uniform distribution, suggesting that M1 modulation is associated with the presence of NEls in the early anticipatory period. $\boldsymbol{B}$, The panel shows the proportion of correct trials occurring after an early -500 to $-250 \mathrm{~ms}$ or late -200 to $0 \mathrm{~ms}$ NEI. The presence of an NEl during the early anticipatory period was associated with a similar proportion of correct aperture discriminations in control, saline, and muscimol conditions (interval -500 to $250 \mathrm{~ms}$; left). However, the presence of an $\mathrm{NEI}$ in the late anticipatory period was associated with a significantly smaller proportion of correct trials after muscimol infusion, when compared to control or saline conditions (interval -200 to $0 \mathrm{~ms}$; right). This result indicates that M1 directly affects NEls in the late anticipatory period, leading to incorrect discrimination trials.

\section{Discussion}

By simultaneously recording the activity of neuronal ensembles in $\mathrm{M} 1, \mathrm{~S} 1, \mathrm{VPM}$, and POM as rats performed an aperture discrimination task, we demonstrated the occurrence of anticipa- tory neuronal activity in all major cortical and thalamic structures that define the multiple TCLs of the rat trigeminal somatosensory system. The presence of prestimulus anticipatory activity has been identified previously in the S1 of rats performing this task (Krupa et al., 2004; Pantoja et al., 2007; Wiest et al., 2010). In the present study, however, we observed for the first time the widespread presence of such anticipatory firing activity in the major thalamic nuclei of both the lemniscal and paralemniscal pathways of the rat trigeminal system. The patterns of anticipatory activity were region and layer specific.

Control experiments revealed that trigeminal ganglion neurons, recorded simultaneously from S1 and VPM neurons, did not exhibit any excitatory sensory-evoked responses during the anticipatory period. Thus, activation of peripheral first-order whisker afferents cannot account for the widespread anticipatory activity observed at cortical and thalamic levels. Additional control experiments obtained from simultaneous EMGs, facial nerve lesions, and thalamic recordings, and high-speed video analysis of whisker movements confirmed categorically that the anticipatory activity observed in the S1, VPM, and POM was not caused by whisker movements or contact with any objects.

The anticipatory activity recorded across the TCLs was comprised of increases and decreases in neuronal activity that occurred in both lemniscal and paralemniscal thalamic relays of the trigeminal system. The timing of the onset of increased anticipatory activity in S1 and thalamic nuclei was linearly related to the animal's overall discrimination performance, which in turn was linearly related to the animal's locomotion speed.

Inactivation of $\mathrm{M} 1$ induced distinct changes in the magnitude and duration of anticipatory neuronal firing modulations across multiple areas. First, M1 inactivation changed the proportions of individual neurons modulated in S1 in a layer-specific fashion. It also reduced the magnitude of anticipatory activity in POM and VPM, and disrupted the duration and the timing of anticipatory activity onset in S1. M1 inactivation resulted in a decrease in the animal's discrimination performance, although no gross motor impairments occurred. However, the onset of S1 anticipatory activity was no longer a good predictor of the performance. Also, M1 modulation of S1 anticipatory activity was associated with different encoding of tactile information at single trials. These results suggest that top-down modulations by $\mathrm{M} 1$ neurons affect the entire somatosensory thalamocortical loop and play an important role in active tactile discrimination.

\section{Anticipatory activity is not related to whisker movements} Our present and previous findings suggest that thalamic and cortical anticipatory activity does not result from whisker or head movements, or from whiskers contacting the surfaces or objects in the recording box. Our extensive control experiments demonstrated that (1) anticipatory S1 and VPM neuronal modulations are present while TG is silent or EMG activity is abolished by facial nerve lesions (Wiest et al., 2010; present study), (2) these cortical modulations appear during training of the active tactile discrimination task and are present even in the absence of the tactile stimulus (Wiest et al., 2010), (3) animals perform well after facial nerve lesions (Krupa et al., 2004), and (4) video analysis repeatedly demonstrated that no whisker contacts or head movements are present as the animal moves between the door and the discrimination bars (Krupa et al., 2004; Wiest et al., 2010; present study). These results rule out peripheral input as an essential contributor to anticipatory cortical and thalamic activity. 


\section{Anticipatory activity is primarily cortically driven}

Our results suggest that M1 significantly contributes to anticipatory activity in multiple TCLs simultaneously. Previous studies in anesthetized animals have shown that pharmacological enhancement of M1 activity facilitates neuronal responses to whisker stimulation in infragranular layers of S1 and VPM (Lee et al., 2008). These authors showed that although responses in $S 1$ and VPM were lower when awake animals were whisking, the inactivation of the nucleus interpolaris increased these responses, suggesting that gating of tactile responses during whisking was mediated, at least partially, by the trigeminal nuclei. As corticofugal cells in M1 do not project to the trigeminal nuclei (Miyashita et al., 1994; Miyashita and Mori, 1995) and the effects of M1 stimulation are abolished after S1 and S2 lesions (Urbain and Deschenes, 2007b), it is likely that the anticipatory effects we observed in multiple cortical and subcortical areas were formed by complex loops involving M1, S1, S2, and the thalamic and trigeminal nuclei (Furuta et al., 2010; Viaene et al., 2011). It is important to stress that in the present study we did not specifically test whether M1 affected anticipatory thalamic activity through corticobulbar loops. This important issue will be addressed by future studies.

\section{Anticipatory activity, speed, and performance}

It is not entirely clear how neurons exhibiting anticipatory activity may be involved in gating during whisking, since animals do not generally whisk during this task (Krupa et al., 2004; Wiest et al., 2010; present study). Yet, based on the results presented here, we hypothesize that anticipatory activity represents a form of motor gating by M1 related to the animal's locomotion speed. Although motor gating has a long history in the somatosensory literature (Chapin and Woodward, 1981, 1982a,b), in the rat trigeminal system it has mostly been attributed to active whisking (Fanselow and Nicolelis, 1999; Fanselow et al., 2001; Nicolelis and Fanselow, 2002). Interestingly, previous reports have shown that trained rats moving at fast speeds do not whisk (Arkley et al., 2011). In our task, the same type of behavioral strategy (high locomotion speed without whisking) may have been used by our subjects. It is likely that different speeds generate different tactile representations of the stimulus and that disruption of such motor gating by muscimol inactivation of M1 would alter the animal's behavioral performance. Anticipatory increases in cortical and thalamic activity (with an origin in M1) end the moment that the tactile stimulus information arrives. We speculate that after inactivation of M1, the onsets and offsets of anticipatory activity are no longer coordinated with the sampling of tactile information. This could in turn disrupt the coordinated activity along multiple thalamocortical structures and diminish the animal's tactile discrimination performance. This hypothesis is in line with our previous findings (Fanselow and Nicolelis, 1999; Fanselow et al., 2001; Nicolelis and Fanselow, 2002).

\section{Trigeminal pathways are not functionally independent}

In this study, we found that the main periods of increased and decreased neural anticipatory activity are shared by multiple cortical areas and thalamic nuclei. The overall patterns of activation described here are in line with the functional roles attributed previously to the lemniscal and paralemniscal pathways. Specifically, the characteristic patterns of activity found in the VPM barreloid core and S1 layer IV both support the previously known role of these regions in active tactile processing (ArmstrongJames and Fox, 1987; Krupa et al., 2004). Also, the overall increases in anticipatory activity present in infragranular layers of
S1, POM (Pierret et al., 2000; Veinante et al., 2000; Furuta et al., 2006; Masri et al., 2008), and the head of the barreloids in the VPM (Urbain and Deschenes, 2007a) suggest that these regions are under the influence of M1, possibly being associated with sensorimotor integration (Urbain and Deschenes, 2007b; Lee et al., 2008; Hill et al., 2011; Petreanu et al., 2012). However, the widespread but specific effects of M1 inactivation found in all thalamic nuclei and regions in the present study clearly indicate that to regard the lemniscal and paralemniscal pathways as parallel and independent processing units would constitute a serious underrepresentation of the true rich physiological cross-talk interactions that take place within these multiple streams of the trigeminal system.

\section{Conclusion}

Our results suggest that the overall state of the thalamocortical neural network, rather than a single cortical or thalamic relay, determines how information is processed following a tactile stimulus presentation. More generally, the results of this and other studies from our laboratory strongly support the asynchronous convergence hypothesis (Nicolelis et al., 1995; Nicolelis, 2005), i.e., that active tactile discrimination results from the dynamic interplay of multiple descending, ascending, and local afferents that converge asynchronously on neurons located at each stage of the trigeminal pathway. Such an arrangement determines the emergence of highly dynamic and distributed spatiotemporal patterns of neuronal ensemble activity in each of these locations. The present results also suggest that before and at the moment of contact with a tactile stimulus, the somatosensory system is already performing a series of preparatory operations that constrain or facilitate the discrimination of the tactile stimulus that is about to touch the facial whiskers. According to the asynchronous hypothesis, modulation of any of the afferents or components of the trigeminal system may affect the overall state of the network and influence tactile processing at all other levels of the system. Accordingly, our present findings frontally challenge the classical labeled line hypothesis (Welker, 1976) that proposes that strict and highly segregated ascending feedforward pathways account for the entire processing of tactile information in the rat trigeminal system.

\section{References}

Arkley K, Grant R, Mitchinson B, Prescott T (2011) The relationship between whisking and locomotion in rats. Soc Neurosci Abstr 37:76.24.

Armstrong-James M, Fox K (1987) Spatiotemporal convergence and divergence in the rat S1 "barrel" cortex. J Comp Neurol 263:265-281. CrossRef Medline

Belford GR, Killackey HP (1979) Vibrissae representation in subcortical trigeminal centers of the neonatal rat. J Comp Neurol 183:305-321. CrossRef Medline

Brecht M, Schneider M, Sakmann B, Margrie TW (2004) Whisker movements evoked by stimulation of single pyramidal cells in rat motor cortex. Nature 427:704-710. CrossRef Medline

Chapin JK, Woodward DJ (1981) Modulation of sensory responsiveness of single somatosensory cortical cells during movement and arousal behaviors. Exp Neurol 72:164-178. CrossRef Medline

Chapin JK, Woodward DJ (1982a) Somatic sensory transmission to the cortex during movement: gating of single cell responses to touch. Exp Neurol 78:654-669. CrossRef Medline

Chapin JK, Woodward DJ (1982b) Somatic sensory transmission to the cortex during movement: phasic modulation over the locomotor step cycle. Exp Neurol 78:670-684. CrossRef Medline

Ego-Stengel V, Le Cam J, Shulz DE (2012) Coding of apparent motion in the thalamic nucleus of the rat vibrissal somatosensory system. J Neurosci 32:3339-3351. CrossRef Medline

Fanselow EE, Nicolelis MA (1999) Behavioral modulation of tactile re- 
sponses in the rat somatosensory system. J Neurosci 19:7603-7616. Medline

Fanselow EE, Sameshima K, Baccala LA, Nicolelis MA (2001) Thalamic bursting in rats during different awake behavioral states. Proc Natl Acad Sci U S A 98:15330-15335. CrossRef Medline

Furuta T, Nakamura K, Deschenes M (2006) Angular tuning bias of vibrissa-responsive cells in the paralemniscal pathway. J Neurosci 26: 10548-10557. CrossRef Medline

Furuta T, Urbain N, Kaneko T, Deschênes M (2010) Corticofugal control of vibrissa-sensitive neurons in the interpolaris nucleus of the trigeminal complex. J Neurosci 30:1832-1838. CrossRef Medline

Ghazanfar AA, Nicolelis MA (1997) Nonlinear processing of tactile information in the thalamocortical loop. J Neurophysiol 78:506-510. Medline

Ghazanfar AA, Nicolelis MA (2001) Feature article: the structure and function of dynamic cortical and thalamic receptive fields. Cereb Cortex 11: 183-193. CrossRef Medline

Ghazanfar AA, Krupa DJ, Nicolelis MA (2001) Role of cortical feedback in the receptive field structure and nonlinear response properties of somatosensory thalamic neurons. Exp Brain Res 141:88-100. CrossRef Medline

Gutierrez R, Carmena JM, Nicolelis MA, Simon SA (2006) Orbitofrontal ensemble activity monitors licking and distinguishes among natural rewards. J Neurophysiol 95:119-133. Medline

Gutierrez R, Simon SA, Nicolelis MA (2010) Licking-induced synchrony in the taste-reward circuit improves cue discrimination during learning. J Neurosci 30:287-303. CrossRef Medline

Hill DN, Curtis JC, Moore JD, Kleinfeld D (2011) Primary motor cortex reports efferent control of vibrissa motion on multiple timescales. Neuron 72:344-356. CrossRef Medline

Krupa DJ, Ghazanfar AA, Nicolelis MA (1999) Immediate thalamic sensory plasticity depends on corticothalamic feedback. Proc Natl Acad Sci U S A 96:8200-8205. CrossRef Medline

Krupa DJ, Matell MS, Brisben AJ, Oliveira LM, Nicolelis MA (2001) Behavioral properties of the trigeminal somatosensory system in rats performing whisker-dependent tactile discriminations. J Neurosci 21:5752-5763. Medline

Krupa DJ, Wiest MC, Shuler MG, Laubach M, Nicolelis MA (2004) Layerspecific somatosensory cortical activation during active tactile discrimination. Science 304:1989-1992. CrossRef Medline

Lee S, Carvell GE, Simons DJ (2008) Motor modulation of afferent somatosensory circuits. Nat Neurosci 11:1430-1438. CrossRef Medline

Martin JH (1991) Autoradiographic estimation of the extent of reversible inactivation produced by microinjection of lidocaine and muscimol in the rat. Neurosci Lett 127:160-164. CrossRef Medline

Masri R, Bezdudnaya T, Trageser JC, Keller A (2008) Encoding of stimulus frequency and sensor motion in the posterior medial thalamic nucleus. J Neurophysiol 100:681-689. CrossRef Medline

Miyashita E, Mori S (1995) The superior colliculus relays signals descending from the vibrissal motor cortex to the facial nerve nucleus in the rat. Neurosci Lett 195:69-71. CrossRef Medline

Miyashita E, Keller A, Asanuma H (1994) Input-output organization of the rat vibrissal motor cortex. Exp Brain Res 99:223-232. Medline

Nicolelis MA (2005) Computing with thalamocortical ensembles during different behavioural states. J Physiol 566:37-47. CrossRef Medline

Nicolelis MA, Chapin JK (1994) Spatiotemporal structure of somatosensory responses of many-neuron ensembles in the rat ventral posterior medial nucleus of the thalamus. J Neurosci 14:3511-3532. Medline

Nicolelis MA, Fanselow EE (2002) Thalamocortical [correction of Thalamcortical] optimization of tactile processing according to behavioral state. Nat Neurosci 5:517-523. CrossRef Medline
Nicolelis MA, Baccala LA, Lin RC, Chapin JK (1995) Sensorimotor encoding by synchronous neural ensemble activity at multiple levels of the somatosensory system. Science 268:1353-1358. CrossRef Medline

Nicolelis MA, Stambaugh CR, Brisben AJ, Laubach M (1999) Methods for simultaneous multisite neural ensemble recordings in behaving primates. In: Methods for neural ensemble recordings (Nicolelis MA, ed), pp 121156. Boca Raton, FL: CRC.

Nicolelis MA, Dimitrov D, Carmena JM, Crist R, Lehew G, Kralik JD, Wise SP (2003) Chronic, multisite, multielectrode recordings in macaque monkeys. Proc Natl Acad Sci U S A 100:11041-11046. CrossRef Medline

Pantoja J, Ribeiro S, Wiest M, Soares E, Gervasoni D, Lemos NA, Nicolelis MA (2007) Neuronal activity in the primary somatosensory thalamocortical loop is modulated by reward contingency during tactile discrimination. J Neurosci 27:10608-10620. CrossRef Medline

Paxinos G, Watson C (1998) The rat brain in stereotaxic coordinates. New York: Academic.

Petreanu L, Gutnisky DA, Huber D, Xu NL, O’Connor DH, Tian L, Looger L, Svoboda K (2012) Activity in motor-sensory projections reveals distributed coding in somatosensation. Nature 489:299-303. CrossRef Medline

Pierret T, Lavallée P, Deschênes M (2000) Parallel streams for the relay of vibrissal information through thalamic barreloids. J Neurosci 20:74557462. Medline

Shuler MG, Krupa DJ, Nicolelis MA (2001) Bilateral integration of whisker information in the primary somatosensory cortex of rats. J Neurosci 21: 5251-5261. Medline

Shuler MG, Krupa DJ, Nicolelis MA (2002) Integration of bilateral whisker stimuli in rats: role of the whisker barrel cortices. Cereb Cortex 12:86-97. CrossRef Medline

Sperry RW (1950) Neural basis of the spontaneous optokinetic response produced by visual inversion. J Comp Physiol Psychol 43:482-489. CrossRef Medline

Urbain N, Deschênes M (2007a) A new thalamic pathway of vibrissal information modulated by the motor cortex. J Neurosci 27:12407-12412. CrossRef Medline

Urbain N, Deschênes M (2007b) Motor cortex gates vibrissal responses in a thalamocortical projection pathway. Neuron 56:714-725. CrossRef Medline

Van Der Loos H (1976) Barreloids in mouse somatosensory thalamus. Neurosci Lett 2:1-6. CrossRef Medline

Veinante P, Lavallée P, Deschênes M (2000) Corticothalamic projections from layer 5 of the vibrissal barrel cortex in the rat. J Comp Neurol 424:197-204. CrossRef Medline

Viaene AN, Petrof I, Sherman SM (2011) Properties of the thalamic projection from the posterior medial nucleus to primary and secondary somatosensory cortices in the mouse. Proc Natl Acad Sci U S A 108:18156-18161. CrossRef Medline

Welker C (1976) Receptive fields of barrels in the somatosensory neocortex of the rat. J Comp Neurol 166:173-189. CrossRef Medline

Wiest MC, Bentley N, Nicolelis MA (2005) Heterogeneous integration of bilateral whisker signals by neurons in primary somatosensory cortex of awake rats. J Neurophysiol 93:2966-2973. CrossRef Medline

Wiest MC, Thomson E, Pantoja J, Nicolelis MA (2010) Changes in S1 neural responses during tactile discrimination learning. J Neurophysiol 104: 300-312. CrossRef Medline

Woolsey TA, Van der Loos H (1970) The structural organization of layer IV in the somatosensory region (SI) of mouse cerebral cortex. The description of a cortical field composed of discrete cytoarchitectonic units. Brain Res 17:205-242. CrossRef Medline 